\title{
Behavioral Mean-Variance Portfolio Selection
}

\author{
Junna Bi* Hanqing $\mathrm{Jin}^{\dagger} \quad$ Qingbin Meng ${ }^{\ddagger}$
}

\begin{abstract}
In this paper, a behavioral mean-variance portfolio selection problem in continuous time is formulated and studied. Unlike in the standard mean-variance portfolio selection problem, the cumulative distribution function of the cash flow is distorted by the probability distortion function used in the behavioral mean-variance portfolio selection problem. In our model, our optimization problem cannot be considered convex because its convexity is destroyed by the probability distortion function. This feature distinguishes our problem from the conventional linear-quadratic (LQ) problems and makes conventional approaches, such as convex optimization, dynamic programming, and the stochastic optimal LQ control theory, unusable in our model. To address this challenge, we propose and demonstrate a solution scheme by taking the quantile function of the terminal cash flow as the decision variable, and then replace the corresponding optimal terminal cash flow with the optimal quantile function. This allows the efficient frontier and the efficient strategy to be exploited.
\end{abstract}

Key words: Applied probability; Behavioural OR; Mean-variance portfolio selection; Probability distortion; Quantile approach.

AMS 2000 Subject Classification: 91B28

\section{Introduction}

The modern portfolio selection theory is a theory of investment that attempts to maximize a portfolio's expected return given a particular amount of portfolio risk. This theory also tries to minimize risk for a given level of expected return by choosing the proportions of various assets that make up the portfolio. Since the fundamental work of Markowitz (1952), the mean-variance optimization problem has become one of the key topics in financial theory and a rather popular criterion to measure risk. The mean-variance portfolio selection problem tries to seek out the best allocation of wealth among a variety of securities so as to achieve the optimal trade-off between the risk over a fixed time horizon and the expected return on investment. Markowitz (1952) provided a fundamental basis for portfolio construction in a single period, in which the risk of a portfolio was measured by the variance of its return and the benefit was measured by the expected return. Markowitz formulates how to minimize a portfolio's variance subject to the constraint in which the expectation of the portfolio equals the prescribed level. Such an optimal portfolio is said to be variance minimizing. If it also achieves the maximum expected return among all portfolios having the same variance of return, then it is said to be an efficient

\footnotetext{
${ }^{*}$ School of Statistics, East China Normal University, Shanghai 200241, P. R. China. E-mail: jnbi@sfs.ecnu.edu.cn.

${ }^{\dagger}$ Mathematical Institute and Oxford-Man Institute of Quantitative Finance, The University of Oxford, Andrew Wiles Building, Woodstock Road, Oxford OX2 6GG. E-mail: jinh@maths.ox.ac.uk.

${ }^{\ddagger}$ Corresponding author. Finance Department, School of Business, Renmin University of China, 100872, P. R. China. E-mail: meng_q_b@126.com.
} 
strategy (or efficient portfolio). The two-dimensional set of points that, in place of variance and expected return, which are produced by efficient portfolios, is called the efficient frontier. After the work of Markowitz (1952), mean-variance criterion has become popular in finance theory for measuring risk and is widely used within main stream literature. See e.g. Merton (1972), Zhou and Li (2000), Li, Zhou and Lim (2002), Bielecki et al. (2005), Markowitz (2014), Cui et al. (2014) and Yao, Li and Li (2016), Dang and Forsyth (2016), Lioui and Poncet (2016), and Ray and Jenamani (2016).

Substantial evidences, such as the Friedman and Savage puzzle (Friedman and Savage 1948), the Allais paradox (Allais 1953), the Ellesberg paradox (Ellesberg 1961), and the equity premium puzzle (Mehra and Prescott 1985), indicate that decision makers do not usually treat probabilities linearly. Specifically, people tend to overestimate small probabilities and underestimate large probabilities. One way to model such distortions in decision making is through a probability weighting function, which uses a nonlinear transformation and applies it to the underlying probability measurement when risky choices are evaluated.

The probability weighting function is introduced by Yarri's "dual theory of choice" (Yarri 1987), which attempts to resolve decision making issues as mentioned above. The main feature of this theory is that an investor will distort the probability cumulative function of the random payment used as the basis of this theory. As Yarri (1987) shows, the probability distortion function takes a different approach in the way it represents risk preference. Moreover, there exist other theories, such as Lopes's SP/A (security-potential/aspiration) model (Lopes 1987, Lopes and Oden 1999) and Kahneman and Tversky's Cumulative Prospect Theory (Kahneman and Tversky 1979, Tversky and Kahneman 1992), that develop with a focus on involving probability distortion. The SP/A model is a dual criterion model that describes the process of choosing between two logically and psychologically separate criteria: security-potential criterion and aspiration criterion. In SP/A theory, a decision maker's choice is based upon an evaluation function of SP (security-potential), which reflects the emotions of fear and hope, along with an aspiration probability A. SP/A theory is regarded as a branch of the behavioral decision-making model. Cumulative Prospect Theory is formulated based on a series of laboratory experiments that model decision making behavior. Cumulative Prospect Theory uses a reference point to define gains (excesses of wealth over the reference point) and losses (shortfalls from the reference point). This theory also defines the utility and dis-utility functions alongside the probability distortions of gains and losses.

Recently, there has been a growing trend of incorporating the probability distortion into portfolio choice, see e.g. Levy and Levy (2004) and Gomes (2005), but these models have been limited to the single-period setting. The reason for that limitation is because in a continuous time portfolio selection model, many conventional and convenient approaches, such as convex optimization, dynamic programming, and stochastic control, cannot be used due to the nonlinear distortion in probabilities. Fortunately, Jin and Zhou (2008) developed a quantile approach to overcome the difficulties arising from the probability distortion. They formulated and studied a general continuous-time behavioral portfolio selection model under Kahneman and Tversky's Cumulative Prospect Theory, featuring S-shaped utility (value) functions and probability distortions. Furthermore, a new portfolio choice model is formulated by He and Zhou (2011) with probability distortion in continuous time for both complete and incomplete markets, in which the quantile function of the terminal cash flow is taken as the decision variable. Their formulation covers a wide body of existing and new models with law-invariant preference measures. Bi et al. (2013) formulated and studied a mean-semivariance portfolio selection problem in continuous time when the probability was distorted by a nonlinear transformation. The optimal solutions are derived by using the quantile approach, which is formulated by Jin and Zhou (2008) and 
developed in He and Zhou (2011). Other works about behavioral finance theory and behavioral operational research can be found in Jin and Zhou (2013), Björk et al. (2014), Cillo and Delquié (2014), Brocklesby (2016), He et al. (2017), and so on.

How do we then deal with the behavioral mean-variance portfolio selection problem, including the probability distortion feature? To our knowledge and according to available literature, little research has formally been done thus far to formulate or resolve this problem. This paper is the first to present a study of the behavioral mean-variance portfolio selection problem.

The main technical challenge of this study is that inclusion of probability distortion (or nonlinear expectation) destroys the models time-consistency, which is necessary for the dynamic programming approach. It also destroys the model's convexity, which is necessary for the convex duality approach. We use the quantile approach to deal with the challenge. In our model, the quantile function of the terminal cash flow becomes the decision variable. After finding the optimal quantile function, the corresponding optimal terminal cash flow can be recovered. We can then obtain the efficient frontier and the efficient strategy, which is the hedging strategy of the optimal terminal cash flow.

We divide our behavioral mean-variance portfolio selection problem into two subproblems. The first subproblem is to find the optimal attainable wealth $X^{*}$, i.e., the random variable that is the optimal value of all possible $x(T)$ generated by admissible portfolios. The second subproblem is to find a tame portfolio $\pi^{*}(\cdot)$ that replicates $X^{*}$, where $X^{*}$ is the solution of the first subproblem if it exists. To solve the first subproblem, we use the quantile approach.

First, we split the first subproblem into positive and negative part problems. Second, due to the probability distortion involved in our model, the feasibility of the first subproblem is no longer definite. Whether or not a feasible solution can be found for this problem is the main question for this part of the subproblem. Third, due to the probability distortion, the positive part problem is a constrained non-convex minimization problem. We turn it into a convex minimization problem by changing the decision variable and performing a series of transformations. Then we use the Lagrange method to solve the convex minimization problem. Using the same method, we then solve the negative part problem. Fourth, the original optimal problem will be solved by merging the positive and negative part problems from step three. Finally, we study typical cases of probability distortion in the financial market, and present their explicit solutions if they exist. Some of the most used probability distortions are included in our cases, and we give their solution schemes if they exist. To solve the second subproblem, the optimal trading strategy of the behavioral mean-variance problem will be expressed by a solution of a backward stochastic differential equation.

The main contribution of this paper has three parts: First, our exploration into an important research area of modern financial theory by incorporating the probability distortion feature into the conventional mean-variance portfolio selection problem while taking into consideration the behavioral mean-variance portfolio selection problem across continuous time. We use the quantile approach to solve our optimization problem. Second, we provide a solution that addresses the feasibility of our model. In the conventional mean-variance portfolio selection model, the feasibility is trivial. When the probability distortion is added to the model, the feasibility becomes nontrivial, but remains challenging to determine because of the constraints imposed by the probability distortion. We provide sufficient and necessary conditions needed to show that there is a feasible solution. Third, we present the optimal solution, when it exists, and then exploit both the efficient frontier and the efficient strategy. Finally, we provide different investment strategies (asset-allocation schemes) for the investors with specific subjective probabilities.

The organization of this paper is as follows: In Section 2, we formulate the behavioral mean-variance portfolio selection problem. Sections 3 and 4 are devoted to solving the first 
subproblem. In Section 3, we derive the optimal terminal wealth via quantiles by solving the positive and negative part problems separately. In Section 4, we give the final solution and some analysis of the optimal solution. In Section 5, we give some specific examples to show how our result is applied, and in Section 6, we solve for the efficient frontier and efficient strategy. Some numerical examples are given to further illustrate our results in Section 7. Finally, Section 8 concludes the paper.

\section{Problem formulation}

In this section, we set up the continuous-time financial market in Subsection 2.1 and formulate our behavioral mean-variance portfolio selection problem in Subsection 2.2. Because our problem is a constrained non-convex minimization problem due to the probability distortion, the standard approaches, such as the convex duality and the dynamic programming, fail to apply. Hence, we turn our problem into a convex minimization problem by changing the decision variable and performing a series of transformations in Subsection 2.3. Then we use the Lagrange method to solve the convex minimization problem in the upcoming sections. Based on some analysis and observations, we split the problem formulated in Subsection 2.3 into positive and negative part problems, the process of which is shown in Subsection 2.4. Due to the probability distortion involved in our model, the feasibilities of problems formulated in Subsection 2.3 and Subsection 2.4 are no longer trivial. The feasibility of these problems is the question we tackle in Subsection 2.5.

\subsection{The financial market}

Let $(\Omega, \mathcal{F}, \mathcal{P})$ be a probability space, with filtration $\left\{\mathcal{F}_{t}\right\}_{t \geq 0}$ containing all objects defined in the following. We suppose that there exists a financial market where $m+1$ assets are traded continuously over a finite time horizon $[0, T]$. The risk-free asset's price process $P_{0}(t)$ is subject to the following differential equation

$$
\left\{\begin{aligned}
d P_{0}(t) & =r(t) P_{0}(t) d t, t \in[0, T], \\
P_{0}(0) & =p_{0},
\end{aligned}\right.
$$

where the interest rate $r(\cdot)$ is an $\mathcal{F}_{t}$-adapted, scalar-valued stochastic process with $\int_{0}^{T}|r(t)| d t<$ $+\infty$ a.s..

The other $m$ assets are risky assets (stocks), and their price processes $P_{i}(t), i=1,2, \cdots, m$, satisfy the following stochastic differential equations

$$
\left\{\begin{array}{l}
d P_{i}(t)=P_{i}(t)\left[b_{i}(t) d t+\sum_{j=1}^{m} \sigma_{i j}(t) d W_{j}(t)\right], \begin{array}{r}
t \in[0, T], \\
P_{i}(0)=p_{i},
\end{array} \quad i=1,2, \cdots, m,
\end{array}\right.
$$

where $W(t):=\left(W_{1}(t), W_{2}(t), \cdots, W_{m}(t)\right)^{\top}$ is a standard $\left\{\mathcal{F}_{t}\right\}_{t \geq 0}$-adapted $m$-dimensional Brownian motion, with the superscript $T$ here and after means the transpose of a matrix or a vector. $b_{i}(t)(>r(t))$ and $\sigma_{i j}(t)$, the appreciation rates and the volatility rates respectively, are scalarvalued, $\mathcal{F}_{t}$-progressively measurable stochastic processes with

$$
\int_{0}^{T}\left[\sum_{i=1}^{m}\left|b_{i}(t)\right|+\sum_{i=1}^{m} \sum_{j=1}^{m} \sigma_{i j}^{2}(t)\right] d t<+\infty \text { a.s.. }
$$


We set the excess rate of return process

$$
B(t):=\left(b_{1}(t)-r(t), \ldots, b_{m}(t)-r(t)\right)^{\top},
$$

and define the volatility matrix process $\sigma(t):=\left(\sigma_{i j}(t)\right)_{m \times m}$. This financial market model has been studied extensively in the literature; see for example, Jin and Zhou (2008), He and Zhou (2011) and Bi et al. (2013). The basic assumptions imposed on the market parameters throughout this paper are the same as Assumption 2.1 in Jin and Zhou (2008) (or Assumption 2.1 in $\mathrm{Bi}$ et al. 2013). Then there exists a unique risk-neutral probability measure $\mathbb{Q}$ defined by $\left.\frac{d \mathbb{Q}}{d \mathbb{P}}\right|_{\mathcal{F}_{t}}=\rho(t)$, where

$$
\rho(t)=\exp \left\{-\int_{0}^{t}\left[r(s)+\frac{1}{2}|\theta(s)|^{2}\right] d s-\int_{0}^{t} \theta(s)^{\top} d W(s)\right\}
$$

is the pricing kernel or state density price. We denote $\rho:=\rho(T)$ with its cumulative distribution function $F_{\rho}(\cdot)$. Note that $0<\rho<+\infty$ a.s. and $0<\mathbb{E} \rho<+\infty$. Moreover, we have the following assumption for $\rho$ in this paper.

Assumption $2.1 \rho$ admits no atom.

Assumption 2.1 is the same as that in Jin and Zhou (2008) (or Bi et al. 2013). This assumption is not essential, but is imposed to avoid undue technicality. In particular, the assumption is satisfied when $r(\cdot)$ and $\theta(\cdot)$ are deterministic with $\int_{0}^{T} \theta^{2}(t) d t \neq 0$, in which case $\rho$ is a non-degenerate lognormal random variable.

Suppose that the agent is allowed to invest all of his/her wealth in the financial market, with an initial wealth $x_{0}>0$ and an investment horizon $[0, T]$. We assume that the trading of shares takes place in a self-financing fashion, i.e., there is no consumption or income. And there is no transaction cost. Let $x(t)$ denote the agent's total wealth at time $t$ and $\pi_{i}(t), i=$ $1,2, \cdots, m$, denote the total market value of the agent's wealth in the $i$-th stock at time $t$. Then $x(t)-\sum_{i=1}^{m} \pi_{i}(t)$ is the value of the agent's wealth in the risk-free asset. Then $x(t)$ satisfies

$$
d x(t)=\frac{x(t)-\sum_{i=1}^{m} \pi_{i}(t)}{P_{0}(t)} d P_{0}(t)+\sum_{i=1}^{m} \frac{\pi_{i}(t)}{P_{i}(t)} d P_{i}(t) .
$$

So $x(\cdot)$ satisfies

$$
\left\{\begin{aligned}
d x(t) & =\left[r(t) x(t)+B(t)^{\top} \pi(t)\right] d t+\pi(t)^{\top} \sigma(t) d W(t), t \in[0, T], \\
x(0) & =x_{0},
\end{aligned}\right.
$$

where $\pi(t):=\left(\pi_{1}(t), \pi_{2}(t), \cdots, \pi_{m}(t)\right)^{\top}$. The process $\pi(\cdot)$ is said to be an admissible portfolio if it is $\mathcal{F}_{t}$-progressively measurable with

$$
\int_{0}^{T}\left|\sigma(t)^{\top} \pi(t)\right|^{2} d t<+\infty \text { and } \int_{0}^{T}\left|B(t)^{\top} \pi(t)\right| d t<+\infty, \text { a.s.. }
$$

\subsection{The behavioral mean-variance problem}

In this subsection we introduce the behavioral mean-variance problem. First of all, we define the probability distortion functions as follows. 
Definition 2.1 Let $w_{+}(\cdot)$ and $w_{-}(\cdot):[0,1] \rightarrow[0,1]$ be the probability distortion functions (or cumulative weighting functions), representing the distortions in probability for the gains and losses respectively. The probability distortion function describes the subjective inflation or deflation of the true probability. Assume that $w_{ \pm}(\cdot)$ are generally non-linear, strictly increasing and differentiable across $(0,1)$, where $w_{ \pm}(0)=0$ and $w_{ \pm}(1)=1$. Thus the distortion preserves the order of the probabilities, and there is no distortion on sure events.

For a random variable $X$ with cumulative distribution function $F_{X}(x)$, its behavioral mean is defined by

$$
\begin{aligned}
\widetilde{\mathbb{E}}(X): & =\widetilde{\mathbb{E}}_{+}\left(X^{+}\right)-\widetilde{\mathbb{E}}_{-}\left(X^{-}\right)=\int_{0}^{+\infty} w_{+}\left(P\left(Y^{+}>y\right)\right) d y-\int_{0}^{+\infty} w_{-}\left(P\left(Y^{-}>y\right)\right) d y \\
& =\int_{0}^{+\infty} x d\left[-w_{+}\left(1-F_{X}(x)\right)\right]+\int_{-\infty}^{0} x d\left[w_{-}\left(F_{X}(x)\right)\right],
\end{aligned}
$$

where the signs $X^{+}$and $X^{-}$, here and after, denote the positive and negative part of $X$, respectively. For any random variable $X$ satisfied $\widetilde{\mathbb{E}}[X-k]=0$, its behavioral variance is defined by

$$
\begin{aligned}
\widetilde{V}(X) & :=\widetilde{\mathbb{E}}_{+}\left\{\left[(X-k)^{+}\right]^{2}\right\}+\widetilde{\mathbb{E}}_{-}\left\{\left[(X-k)^{-}\right]^{2}\right\} \\
& =\int_{k}^{+\infty}(x-k)^{2} d\left[-w_{+}\left(1-F_{X}(x)\right)\right]+\int_{-\infty}^{k}(x-k)^{2} d\left[w_{-}\left(F_{X}(x)\right)\right]
\end{aligned}
$$

Note that if we put $w_{ \pm}(z)=z$, the behavioral mean and variance of $X$ return to the conventional mean and variance of $X$.

We designate $\mathcal{L}_{\mathcal{F}}^{2}\left(0, T ; \mathbb{R}^{m}\right)$ as the set of all $\mathbb{R}^{m}$-valued, measurable stochastic processes $f(\cdot)=\{f(t): 0 \leq t \leq T\}$, which is adapted to $\left\{\mathcal{F}_{t}\right\}_{t \geq 0}$ such that $\mathbb{E} \int_{0}^{T}|f(t)|^{2} d t<+\infty$. We denote by $\mathcal{L}_{\mathcal{F}_{T}}^{2}\left(\Omega ; \mathbb{R}^{m}\right)$ the set of all $\mathbb{R}^{m}$-valued, $\mathcal{F}_{T}$-measurable random variables $X$ such that $\mathbb{E}|X|^{2}<+\infty$.

The problem we consider in this paper is

$$
\begin{aligned}
& \min \widetilde{V}[x(T)]=\widetilde{\mathbb{E}}_{+}\left\{\left[(x(T)-k)^{+}\right]^{2}\right\}+\widetilde{\mathbb{E}}_{-}\left\{\left[(x(T)-k)^{-}\right]^{2}\right\} \\
& \text { subject to }\left\{\begin{array}{l}
\widetilde{\mathbb{E}}[x(T)-k]=0 \\
\pi(\cdot) \in \mathcal{L}_{\mathcal{F}}^{2}\left(0, T ; \mathbb{R}^{m}\right) \\
(x(\cdot), \pi(\cdot)) \quad \text { satisfy equation }(2.1),
\end{array}\right.
\end{aligned}
$$

where $k$ stands for the expected terminal wealth target, and $\widetilde{V}[x(T)]$ is the behavioral variance of $x(T)$.

Assumption $2.2 x_{0} \leq k \mathbb{E}(\rho)$.

Assumption 2.2 is the same as that in Jin and Zhou (2008) (or Bi et al. 2013). The financial meaning of this assumption is that the investor expects higher terminal wealth $k$ by investing in the stock market than the terminal wealth $\frac{x_{0}}{\mathbb{E}(\rho)}$ of only investing in the risk-free bond market. This assumption also implies that the investor has to take risk to meet his investment target. Clearly, this is a reasonable assumption.

In order to solve problem (2.2), we introduce a proposition, taken from Karoui et al. (1997) that will significantly simplify our problem. 
Proposition 2.1 For any $\xi \in \mathcal{L}_{\mathcal{F}_{T}}^{2}(\Omega ; \mathbb{R})$, the following backward stochastic differential equation

$$
\left\{\begin{array}{l}
d x(t)=\left[r(t) x(t)+\theta(t)^{\top} Z(t)\right] d t+Z(t)^{\top} d W(t), t \in[0, T], \\
x(T)=\xi
\end{array}\right.
$$

admits a unique solution $(x(\cdot), Z(\cdot))$ and $x(t)$ is given by

$$
x(t)=\rho(t) \mathbb{E}\left[\rho(T) \xi \mid \mathcal{F}_{t}\right], \quad \forall t \in[0, T], \text { a.s.. }
$$

In view of Proposition 2.1, we need only solve the following two subproblems. The first subproblem is to find the optimal attainable wealth $X^{*}$, i.e., the random variable that is the optimal value of all possible $x(T)$ generated by admissible portfolios. This subproblem can be represented by

$$
\begin{aligned}
& \min \tilde{V}[X]=\widetilde{\mathbb{E}}_{+}\left\{\left[(X-k)^{+}\right]^{2}\right\}+\widetilde{\mathbb{E}}_{-}\left\{\left[(X-k)^{-}\right]^{2}\right\} \\
& \text { subject to }\left\{\begin{array}{l}
(\widetilde{\mathbb{E}}[X-k]=0 \\
\mathbb{E}[\rho X]=x_{0} \\
X \in \mathcal{L}_{\mathcal{F}}^{2}\left(0, T ; \mathbb{R}^{m}\right), \text { a.s.. }
\end{array}\right.
\end{aligned}
$$

The second subproblem is to find a portfolio $\pi(\cdot)$ that replicates $X^{*}$, where $X^{*}$ is the solution of the first subproblem, and $(x(\cdot), \pi(\cdot))$ satisfy

$$
\left\{\begin{array}{l}
d x(t)=\left[r(t) x(t)+B(t)^{\top} \pi(t)\right] d t+\pi(t)^{\top} \sigma(t) d W(t), t \in[0, T] \\
x(T)=X^{*}
\end{array}\right.
$$

In the following, we solve for both subproblems.

\subsection{Problem formulation via quantile}

For a convex optimization problem, the method of Lagrange multipliers is a powerful tool for finding the maximum and minimum of a function that is subject to equality constraints. By introducing Lagrange multipliers, the constraint conditions can be eliminated. But problem (2.5) is not a convex optimization problem, so the usual Lagrange multiplier method cannot be applied directly. In this subsection, we reformulate this problem via the quantile approach by changing the decision variable and performing a series of transformations. This procedure turns the problem into a convex minimization problem, which will allow us to use the Lagrange method to find a solution to the convex minimization problem. This process will be shown in an upcoming section. To start the process, set $Y:=X-k$, so that the first subproblem (2.5) becomes

$$
\begin{aligned}
& \min \tilde{V}[Y]=\widetilde{\mathbb{E}}_{+}\left[\left(Y^{+}\right)^{2}\right]+\widetilde{\mathbb{E}}_{-}\left[\left(Y^{-}\right)^{2}\right] \\
& \text { subject to }\left\{\begin{array}{l}
\widetilde{\mathbb{E}} Y=0 \\
\mathbb{E}[\rho Y]=y_{0}:=x_{0}-k \mathbb{E}(\rho) \leq 0 \\
Y \in \mathcal{L}_{\mathcal{F}}^{2}\left(0, T ; \mathbb{R}^{m}\right) .
\end{array}\right.
\end{aligned}
$$

Note that if $x_{0}=k \mathbb{E}(\rho)$, and $y_{0}=0$, the optimal solution for problem (2.7) is $Y \equiv 0$. Because of this, we only consider the case of $y_{0}<0$ in the following procedures. 
We change the decision variable from $Y$ to its quantile function, and then turn the above problem into a convex problem through some transformations. For any cumulative distribution functions $F(\cdot)$, we denote its left-inverse function with $F^{-1}(\cdot)$, i.e.,

$$
F_{Y}^{-1}(t)=\inf \left\{x \in \mathbb{R}: F_{Y}(x) \geq t\right\}=\sup \left\{x \in \mathbb{R}: F_{Y}(x)<t\right\}, t \in[0,1] .
$$

Next, we give the following two crucial lemmas, without proof, that we take from Jin and Zhou (2008, Theorem B.1 and Theorem C.1) and Bi et al. (2013, Lemma 3.1 and Lemma 3.2).

Lemma 2.1 Under Assumption 2.1, we have $\mathbb{E}\left[\rho F_{Y}^{-1}\left(1-F_{\rho}(\rho)\right)\right] \leq \mathbb{E}[\rho Y]$, where $F_{Y}(\cdot)$ is the cumulative distribution function of $Y$. Furthermore, if $\mathbb{E}\left[\rho F_{Y}^{-1}\left(1-F_{\rho}(\rho)\right)\right]<\infty$, then the inequality becomes equality if and only if $Y=F_{Y}^{-1}\left(1-F_{\rho}(\rho)\right)$.

Lemma 2.2 If problem (2.7) admits an optimal solution $Y^{*}$ whose distribution function is $F_{Y}(\cdot)$, then $Y^{*}=F_{Y}^{-1}\left(1-F_{\rho}(\rho)\right)$, a.s..

Remark 2.1 Lemma 2.2 shows that an optimal solution $Y^{*}$ of problem (2.7) must be in the form $G\left(Z_{\rho}\right):=F_{Y}^{-1}\left(1-F_{\rho}(\rho)\right)$, where $G(\cdot):=F_{Y}^{-1}(\cdot)$ is the quantile function of $Y^{*}$ and $Z_{\rho}:=$ $1-F_{\rho}(\rho)$. Because $\rho$ is atom-less (Assumption 2.1), we have $Z_{\rho} \sim U(0,1)$, i.e., $Z_{\rho} \sim U(0,1)$ is a particular uniform random variable. In other words, to find an optimal solution $Y^{*}$ (the optimal terminal wealth) of problem (2.7), we need only to search among the random variables of the form $G(\cdot) \in \mathbb{G}$ where $\mathbb{G}$ is the set of all the following quantile functions $F^{-1}(\cdot)$, i.e.,

$$
\mathbb{G}=\left\{G(\cdot):[0,1] \rightarrow \mathbb{R}, \text { non-decreasing, left-continuous, } G(0)=-\infty, G(1):=G\left(1^{-}\right)\right\} .
$$

In addition, we define

$$
\begin{aligned}
& \mathbb{G}_{+}:=\{G(\cdot):[0,1] \rightarrow \mathbb{R}, \text { non-decreasing, left-continuous, } G(z) \geq 0, z \in[0,1]\}, \\
& \mathbb{G}_{-}:=\{G(\cdot):[0,1] \rightarrow \mathbb{R}, \text { non-increasing, left-continuous, } G(z) \geq 0, z \in[0,1]\} .
\end{aligned}
$$

Letting $z=F_{Y}(x)$, we have the following transformation occurs

$$
\begin{aligned}
\widetilde{\mathbb{E}}(Y) & =\mathbb{E}\left[G\left(Z_{\rho}\right)^{+} w_{+}^{\prime}\left(1-Z_{\rho}\right)\right]-\mathbb{E}\left[G\left(Z_{\rho}\right)^{-} w_{-}^{\prime}\left(Z_{\rho}\right)\right] \\
& =\int_{z_{1}}^{1} G(z) w_{+}^{\prime}(1-z) d z+\int_{0}^{z_{1}} G(z) w_{-}^{\prime}(z) d z, \\
\widetilde{V}(Y) & =\widetilde{\mathbb{E}}_{+}\left[Y_{+}^{2}\right]+\widetilde{\mathbb{E}}_{-}\left[Y_{-}^{2}\right]=\mathbb{E}\left\{\left[G\left(Z_{\rho}\right)^{+}\right]^{2} w_{+}^{\prime}\left(1-Z_{\rho}\right)\right\}+\mathbb{E}\left\{\left[G\left(Z_{\rho}\right)^{-}\right]^{2} w_{-}^{\prime}\left(Z_{\rho}\right)\right\} \\
& =\int_{z_{1}}^{1} G(z)^{2} w_{+}^{\prime}(1-z) d z+\int_{0}^{z_{1}} G(z)^{2} w_{-}^{\prime}(z) d z,
\end{aligned}
$$

where $z_{1}=\sup \{z: G(z)<0\}$, and the budget constraint is

$$
\mathbb{E}\left[F_{\rho}^{-1}\left(1-Z_{\rho}\right) G\left(Z_{\rho}\right)\right]=y_{0} .
$$

$w^{\prime}(\cdot)$, here and after, represents the derivative of the function $w(\cdot)$. Then we reformulate our behavioral mean-variance problem via quantiles as follows

$$
\begin{aligned}
& \min \mathbb{E}\left\{\left[G\left(Z_{\rho}\right)^{+}\right]^{2} w_{+}^{\prime}\left(1-Z_{\rho}\right)\right\}+\mathbb{E}\left\{\left[G\left(Z_{\rho}\right)^{-}\right]^{2} w_{-}^{\prime}\left(Z_{\rho}\right)\right\} \\
& \text { subject to }\left\{\begin{array}{l}
\mathbb{E}\left[G\left(Z_{\rho}\right)^{+} w_{+}^{\prime}\left(1-Z_{\rho}\right)\right]-\mathbb{E}\left[G\left(Z_{\rho}\right)^{-} w_{-}^{\prime}\left(Z_{\rho}\right)\right]=0 \\
\mathbb{E}\left[F_{\rho}^{-1}\left(1-Z_{\rho}\right) G\left(Z_{\rho}\right)\right]=y_{0} \\
G(\cdot) \in \mathbb{G} .
\end{array}\right.
\end{aligned}
$$


For convenience, we call problem (2.8) the "quantile problem" in the following sections. The integral version of problem $(2.8)$ is

$$
\begin{aligned}
& \min \int_{z_{1}}^{1} G(z)^{2} w_{+}^{\prime}(1-z) d z+\int_{0}^{z_{1}} G(z)^{2} w_{-}^{\prime}(z) d z \\
& \text { subject to }\left\{\begin{array}{l}
\int_{z_{1}}^{1} G(z) w_{+}^{\prime}(1-z) d z+\int_{0}^{z_{1}} G(z) w_{-}^{\prime}(z) d z=0 \\
\int_{0}^{1} F_{\rho}^{-1}(1-z) G(z) d z=y_{0} \\
G(\cdot) \in \mathbb{G}, z_{1}=\sup \{z: G(z)<0\}
\end{array}\right.
\end{aligned}
$$

Once we have the optimal solution for problem $(2.8), G^{*}(\cdot)$, the optimal solution for $(2.7)$ (the optimal terminal wealth) can be recovered by $Y^{*}=G^{*}\left(1-F_{\rho}(\rho)\right)$. Then, the optimal portfolio can be found using Proposition 2.1. Next, we consider problem (2.8).

\subsection{Splitting into positive part and negative part}

The key idea developed in this paper is taken from Jin and Zhou (2008) which involves splitting the quantile problem (2.8) into positive and negative part problems, and then appropriately merging them. This idea is based on the following observation: If $G(z)$ is a feasible solution of problem (2.8), then we can split it into $G(\cdot)^{+}$and $G(\cdot)^{-}$. We prove the above observation in Subsection 2.5. Furthermore, if $G^{*}(\cdot)$ is optimal for problem $(2.8),\left(G^{*}(\cdot)\right)^{+}$and $\left(G^{*}(\cdot)\right)^{-}$ are respectively optimal for the positive and the negative part problems, with some suitable parameters. We prove this in Theorem 2.1 which is found in this subsection.

In the following steps, we split the quantile problem (2.8) into the positive and negative part problems from $z_{1}$. The behavioral terminal mean and initial price of the positive part are $a_{+}>0$ and $y_{+}>0$, respectively. The behavioral terminal mean and initial price of the negative part are $a_{+}$and $y_{+}-y_{0}$, respectively. An optimal solution for (2.8) should induce the best $\left(z_{1}, a_{+}, y_{+}\right)$. We now carry out this idea in the following two steps.

Step 1: In this step, we consider the positive and negative part problems respectively.

Positive part problem

$$
\begin{aligned}
& \min \mathbb{E}\left[G_{+}\left(Z_{\rho}\right)^{2} w_{+}^{\prime}\left(1-Z_{\rho}\right)\right] \\
& \text { subject to }\left\{\begin{array}{l}
\mathbb{E}\left[G_{+}\left(Z_{\rho}\right) w_{+}^{\prime}\left(1-Z_{\rho}\right)\right]=a_{+} \\
\mathbb{E}\left[G_{+}\left(Z_{\rho}\right) F_{\rho}^{-1}\left(1-Z_{\rho}\right)\right]=y_{+} \\
G_{+}(\cdot) \in \mathbb{G}_{+}, \text {and } G_{+}(z)=0, z \in\left(0, z_{1}\right),
\end{array}\right.
\end{aligned}
$$

where $a_{+}>0, y_{+}>0$. The optimal value of the positive part is denoted by $v_{+}\left(z_{1}, a_{+}, y_{+}\right)$.

Negative part problem

$$
\begin{aligned}
& \min \mathbb{E}\left[G_{-}\left(Z_{\rho}\right)^{2} w_{-}^{\prime}\left(Z_{\rho}\right)\right] \\
& \text { subject to }\left\{\begin{array}{l}
\mathbb{E}\left[G_{-}\left(Z_{\rho}\right) w_{-}^{\prime}\left(Z_{\rho}\right)\right]=a_{+} \\
\mathbb{E}\left[G_{-}\left(Z_{\rho}\right) F_{\rho}^{-1}\left(1-Z_{\rho}\right)\right]=y_{+}-y_{0} \\
G_{-}(\cdot) \in \mathbb{G}_{-}, \text {and } G_{-}(z)=0, z \in\left(z_{1}, 1\right) .
\end{array}\right.
\end{aligned}
$$

The optimal value of the negative part is denoted by $v_{-}\left(z_{1}, a_{+}, y_{+}\right)$. 
The integral version of the positive part is

$$
\begin{aligned}
& \min \int_{z_{1}}^{1} G_{+}(z)^{2} w_{+}^{\prime}(1-z) d z \\
& \text { subject to }\left\{\begin{array}{l}
\int_{z_{1}}^{1} G_{+}(z) w_{+}^{\prime}(1-z) d z=a_{+} \\
\int_{z_{1}}^{1} F_{\rho}^{-1}(1-z) G_{+}(z) d z=y_{+} \\
G_{+}(\cdot) \in \mathbb{G}_{+} .
\end{array}\right.
\end{aligned}
$$

The integral version of the negative part is

$$
\begin{aligned}
& \min \int_{0}^{z_{1}} G_{-}(z)^{2} w_{-}^{\prime}(z) d z \\
& \text { subject to }\left\{\begin{array}{l}
\int_{0}^{z_{1}} G_{-}(z) w_{-}^{\prime}(z) d z=a_{+} \\
\int_{0}^{z_{1}} F_{\rho}^{-1}(1-z) G_{-}(z) d z=y_{+}-y_{0} \\
G_{-}(\cdot) \in \mathbb{G}_{-} .
\end{array}\right.
\end{aligned}
$$

Step 2: In this step, we merge the positive and the negative part problems, and solve for the merged problem.

To start, the resulting problem we get once the positive and negative part problems are merged is as follows,

$$
\begin{aligned}
& \min v_{+}\left(z_{1}, a_{+}, y_{+}\right)+v_{-}\left(z_{1}, a_{+}, y_{+}\right) \\
& \text {subject to }\left\{\begin{array}{l}
z_{1} \in(0,1) \\
a_{+}>0 \\
y_{+}>0
\end{array}\right.
\end{aligned}
$$

The following theorem shows that our problem (2.8) is equivalent to the set of problems (2.9), (2.10) and (2.13). Moreover, the solution of (2.8) can be obtained via the solutions to (2.9), (2.10) and (2.13).

Theorem 2.1 Given $G^{*}(\cdot)$, define

$$
z_{1}^{*}=\sup \left\{z: G^{*}(z)<0\right\}, a_{+}^{*}=\mathbb{E}\left\{\left[G\left(Z_{\rho}\right)^{+}\right]^{2} w_{+}^{\prime}\left(1-Z_{\rho}\right)\right\}
$$

and

$$
y_{+}^{*}=\mathbb{E}\left\{\left[G^{*}\left(Z_{\rho}\right)\right]^{+} F_{\rho}^{-1}\left(1-Z_{\rho}\right)\right\} .
$$

Then $G^{*}(\cdot)$ is optimal for problem (2.8) if and only if the parameters $\left(z_{1}^{*}, a_{+}^{*}, y_{+}^{*}\right)$ are optimal for problem (2.13), and $\left(G^{*}(\cdot)\right)^{+}$as well as $\left(G^{*}(\cdot)\right)^{-}$are respectively optimal for problem (2.9) and (2.10) with parameters $\left(z_{1}^{*}, a_{+}^{*}, y_{+}^{*}\right)$.

Proof. We first prove the "if" part. Assume $\left(G^{*}(\cdot)\right)^{+}$and $\left(G^{*}(\cdot)\right)^{-}$are respectively optimal for problems (2.9) and (2.10) with the parameters $\left(z_{1}^{*}, a_{+}^{*}, y_{+}^{*}\right)$ and $\left(z_{1}^{*}, a_{+}^{*}, y_{+}^{*}\right)$ are optimal for problem (2.13). 
For any feasible solution $G(\cdot)$ of problem $(2.8)$, define $a_{+}:=\mathbb{E}\left[G\left(Z_{\rho}\right)^{+} w_{+}^{\prime}\left(1-Z_{\rho}\right)\right], y_{+}:=$ $\mathbb{E}\left[F_{\rho}^{-1}\left(1-Z_{\rho}\right) G\left(Z_{\rho}\right)^{+}\right]$, and $z_{1}:=\sup \{z: G(z)<0\}$. Therefore, we have $\widetilde{V}\left(G(\cdot)^{+}\right) \geq$ $v_{+}\left(z_{1}, a_{+}, y_{+}\right)$and $\widetilde{V}\left(G(\cdot)^{-}\right) \geq v_{-}\left(z_{1}, a_{+}, y_{+}\right)$. So

$$
\begin{aligned}
\tilde{V}(G(\cdot)) & =\widetilde{V}\left(G(\cdot)^{+}\right)+\widetilde{V}\left(G(\cdot)^{-}\right) \geq v_{+}\left(z_{1}, a_{+}, y_{+}\right)+v_{-}\left(z_{1}, a_{+}, y_{+}\right) \\
& \geq v_{+}\left(z_{1}^{*}, a_{+}^{*}, y_{+}^{*}\right)+v_{-}\left(z_{1}^{*}, a_{+}^{*}, y_{+}^{*}\right)=\widetilde{V}\left(G^{*}(\cdot)\right),
\end{aligned}
$$

which means $G^{*}(\cdot)$ is optimal for $(2.8)$.

For the "only if" part, let $G^{*}(\cdot)$ be optimal for $(2.8)$. It is easy to see $\widetilde{V}\left(G^{*}(\cdot)^{+}\right) \geq$ $v_{+}\left(z_{1}^{*}, a_{+}^{*}, y_{+}^{*}\right)$ and $\widetilde{V}\left(G^{*}(\cdot)^{-}\right) \geq v_{-}\left(z_{1}^{*}, a_{+}^{*}, y_{+}^{*}\right)$. If the former holds true, then there exists a $G_{1}(\cdot)$ which is feasible for $(2.9)$ with parameters $\left(z_{1}^{*}, a_{+}^{*}, y_{+}^{*}\right)$ such that $\widetilde{V}\left(G_{1}(\cdot)\right)<\widetilde{V}\left(G^{*}(\cdot)^{+}\right)$. Then $\bar{G}(\cdot):=G_{1}(\cdot)^{+}+G^{*}(\cdot)^{-}$is feasible for $(2.8)$ and $\widetilde{V}(\bar{G}(\cdot))<\widetilde{V}\left(G^{*}(\cdot)\right)$, which contradicts the optimality of $G^{*}(\cdot)$. So $G^{*}(\cdot)^{+}$is optimal for $(2.9)$. Similarly we can prove that $G^{*}(\cdot)^{-}$is optimal for $(2.10)$. Therefore $\widetilde{V}\left(G^{*}(\cdot)^{+}\right)=v_{+}\left(z_{1}^{*}, a_{+}^{*}, y_{+}^{*}\right)$ and $\widetilde{V}\left(G^{*}(\cdot)^{-}\right)=v_{-}\left(z_{1}^{*}, a_{+}^{*}, y_{+}^{*}\right)$.

Next we show that

$$
v_{+}\left(z_{1}, a_{+}, y_{+}\right)+v_{-}\left(z_{1}, a_{+}, y_{+}\right) \geq v_{+}\left(z_{1}^{*}, a_{+}^{*}, y_{+}^{*}\right)+v_{-}\left(z_{1}^{*}, a_{+}^{*}, y_{+}^{*}\right)=\widetilde{V}\left(G^{*}(\cdot)\right)
$$

for any feasible pair $\left(z_{1}, a_{+}, y_{+}\right)$of problem (2.13).

For any $a_{+}>0, y_{+}>0$, both (2.9) and (2.10) with the parameters $\left(z_{1}, a_{+}, y_{+}\right)$have nonempty feasible regions. Hence for any $\epsilon>0$ there exist $G_{+}(\cdot)$ and $G_{-}(\cdot)$, feasible for (2.9) and (2.10) respectively, such that $\widetilde{V}\left(G_{+}(\cdot)\right)<v_{+}\left(z_{1}, a_{+}, y_{+}\right)+\epsilon$ and $\widetilde{V}\left(G_{-}(\cdot)\right)<v_{-}\left(z_{1}, a_{+}, y_{+}\right)+\epsilon$. We then set $G(\cdot):=G_{+}(\cdot)-G_{-}(\cdot)$, which is feasible for $(2.8)$, and we get

$$
v_{+}\left(z_{1}, a_{+}, y_{+}\right)+v_{-}\left(z_{1}, a_{+}, y_{+}\right)>\widetilde{V}\left(G_{+}(\cdot)\right)+\widetilde{V}\left(G_{-}(\cdot)\right)-2 \epsilon \geq \widetilde{V}\left(G^{*}(\cdot)\right)+2 \epsilon .
$$

At this point, the proof is completed.

\subsection{The feasibility of problems (2.8), (2.9) and (2.10)}

Owing to the probability distortion involved in our model, the feasibilities of the quantile problem (2.8), the positive part problem (2.9) and the negative part problem (2.10) are no longer trivial. Determining the feasibility of solutions for both part problems is the focus of this section.

Proposition 2.2 The feasibility of the quantile problem (2.8) is equivalent to the existence of parameters $\left(z_{1}, a_{+}, y_{+}\right)$for which the positive part problem (2.9) and negative part problem (2.10) are feasible.

Proof. We first assume that there exists a feasible solution $G(z)$ of problem (2.8), where $G(z)$ satisfies

$$
\left\{\begin{array}{l}
\mathbb{E}\left[G\left(Z_{\rho}\right)^{+} w_{+}^{\prime}\left(1-Z_{\rho}\right)\right]-\mathbb{E}\left[G\left(Z_{\rho}\right)^{-} w_{-}^{\prime}\left(Z_{\rho}\right)\right]=0 \\
\mathbb{E}\left[F_{\rho}^{-1}\left(1-Z_{\rho}\right) G^{+}\left(Z_{\rho}\right)\right]-\mathbb{E}\left[F_{\rho}^{-1}\left(1-Z_{\rho}\right) G^{-}\left(Z_{\rho}\right)\right]=y_{0}<0 .
\end{array}\right.
$$

If $\mathbb{E}\left[G\left(Z_{\rho}\right)^{+} w_{+}^{\prime}\left(1-Z_{\rho}\right)\right]=0$, then $\mathbb{E}\left[G\left(Z_{\rho}\right)^{-} w_{-}^{\prime}\left(Z_{\rho}\right)\right]=0$. Thus $G(z)^{+}=G(z)^{-}=0$, a.s., because of $w_{+}^{\prime}(1-z)>0$ and $w_{-}^{\prime}(z)>0$. Consequently,

$$
\mathbb{E}\left[F_{\rho}^{-1}\left(1-Z_{\rho}\right) G\left(Z_{\rho}\right)^{+}\right]-\mathbb{E}\left[F_{\rho}^{-1}\left(1-Z_{\rho}\right) G\left(Z_{\rho}\right)^{-}\right]=0 .
$$


So, we have $\mathbb{E}\left[G\left(Z_{\rho}\right)^{+} w_{+}^{\prime}\left(1-Z_{\rho}\right)\right]>0$. Similarly, we can obtain $\mathbb{E}\left[F_{\rho}^{-1}\left(1-Z_{\rho}\right) G\left(Z_{\rho}\right)^{+}\right]>0$. Set

$$
\begin{aligned}
& z_{1}=\sup \{z: G(z)<0\} \\
& a_{+}:=\mathbb{E}\left[G\left(Z_{\rho}\right)^{+} w_{+}^{\prime}\left(1-Z_{\rho}\right)\right], \\
& y_{+}:=\mathbb{E}\left[F_{\rho}^{-1}\left(1-Z_{\rho}\right) G\left(Z_{\rho}\right)^{+}\right],
\end{aligned}
$$

then $G(\cdot)^{+}$and $G(\cdot)^{-}$are feasible for problem (2.9) and problem (2.10), respectively, with the parameters $\left(z_{1}, a_{+}, y_{+}\right)$.

Conversely, if $G_{+}(\cdot)$ and $G_{-}(\cdot)$ are feasible for problem (2.9) and problem (2.10), respectively, set $G(\cdot)^{+}:=G_{+}(\cdot)$ and $G(\cdot)^{-}:=G_{-}(\cdot)$, then $G(\cdot):=G(\cdot)^{+}-G(\cdot)^{-}$is feasible for problem (2.8). Thus, we get the result.

Next, we solve for the feasibility of the positive part problem. Once that is done, the feasibility of the negative part problem can be derived as well. Define

$$
\begin{gathered}
M_{+}(z):=\frac{F_{\rho}^{-1}(1-z)}{w_{+}^{\prime}(1-z)}, M_{-}(z):=\frac{F_{\rho}^{-1}(1-z)}{w_{-}^{\prime}(z)}, \\
\mathbb{E}_{+}^{z_{1}}(\rho):=\frac{\int_{z_{1}}^{1} F_{\rho}^{-1}(1-z) d z}{\int_{z_{1}}^{1} w_{+}^{\prime}(1-z) d z}=\frac{\int_{z_{1}}^{1} M_{+}(z) w_{+}^{\prime}(1-z) d z}{\int_{z_{1}}^{1} w_{+}^{\prime}(1-z) d z}=\frac{\int_{z_{1}}^{1} M_{+}(z) d\left[-w_{+}(1-z)\right]}{\int_{z_{1}}^{1} d\left[-w_{+}(1-z)\right]}, \\
\mathbb{E}_{-}^{z_{1}}(\rho):=\frac{\int_{0}^{z_{1}} F_{\rho}^{-1}(1-z) d z}{\int_{0}^{z_{1}} w_{-}^{\prime}(z) d z}=\frac{\int_{0}^{z_{1}} M_{-}(z) w_{-}^{\prime}(z) d z}{\int_{0}^{z_{1}} w_{-}^{\prime}(z) d z}
\end{gathered}
$$

and

$$
\begin{aligned}
& \underline{b}:=\inf _{G_{+} \in \mathbb{G}_{+}} \frac{\int_{z_{1}}^{1} G_{+}(z) M_{+}(z) d\left[-w_{+}(1-z)\right]}{\int_{z_{1}}^{1} G_{+}(z) d\left[-w_{+}(1-z)\right]}, \\
& \bar{b}:=\sup _{G_{+} \in \mathbb{G}_{+}} \frac{\int_{z_{1}}^{1} G_{+}(z) M_{+}(z) d\left[-w_{+}(1-z)\right]}{\int_{z_{1}}^{1} G_{+}(z) d\left[-w_{+}(1-z)\right]} .
\end{aligned}
$$

It is easy to prove that $\underline{b} \leq \mathbb{E}_{+}^{z_{1}}(\rho) \leq \bar{b}$. In fact, set $G_{+}(z) \equiv c$, then

$$
\mathbb{E}_{+}^{z_{1}}(\rho)=\frac{\int_{z_{1}}^{1} c M_{+}(z) d\left[-w_{+}(1-z)\right]}{\int_{z_{1}}^{1} c d\left[-w_{+}(1-z)\right]} \in[\underline{b}, \bar{b}] .
$$

Proposition 2.3 If $\underline{b}<\frac{y_{+}}{a_{+}}<\bar{b}$, there exists a feasible solution to problem (2.11). Conversely, if problem (2.11) has a feasible solution, then $\underline{b} \leq \frac{y_{+}}{a_{+}} \leq \bar{b}$.

Proof. For the first part of the proposition, if $\underline{b}<\frac{y_{+}}{a_{+}}<\bar{b}$, there exists $G_{+}^{1}(z) \in \mathbb{G}_{+}, G_{+}^{2}(z) \in$ $\mathbb{G}_{+}$, such that

$$
\frac{\int_{z_{1}}^{1} G_{+}^{1}(z) M_{+}(z) d\left[-w_{+}(1-z)\right]}{\int_{z_{1}}^{1} G_{+}^{1}(z) d\left[-w_{+}(1-z)\right]}<\frac{y_{+}}{a_{+}}<\frac{\int_{z_{1}}^{1} G_{+}^{2}(z) M_{+}(z) d\left[-w_{+}(1-z)\right]}{\int_{z_{1}}^{1} G_{+}^{2}(z) d\left[-w_{+}(1-z)\right]} .
$$

Define

$$
f(\lambda)=\frac{\int_{z_{1}}^{1}\left[\lambda G_{+}^{1}(z)+(1-\lambda) G_{+}^{2}(z)\right] M_{+}(z) d\left[-w_{+}(1-z)\right]}{\int_{z_{1}}^{1}\left[\lambda G_{+}^{1}(z)+(1-\lambda) G_{+}^{2}(z)\right] d\left[-w_{+}(1-z)\right]},
$$


then $f(1)<\frac{y_{+}}{a_{+}}<f(0)$, and $f(\lambda)$ is continuous, so there exists $\lambda_{0} \in(0,1)$, such that

$$
\frac{y_{+}}{a_{+}}=f\left(\lambda_{0}\right)=\frac{\int_{z_{1}}^{1}\left[\lambda_{0} G_{+}^{1}(z)+\left(1-\lambda_{0}\right) G_{+}^{2}(z)\right] M_{+}(z) d\left[-w_{+}(1-z)\right]}{\int_{z_{1}}^{1}\left[\lambda_{0} G_{+}^{1}(z)+\left(1-\lambda_{0}\right) G_{+}^{2}(z)\right] d\left[-w_{+}(1-z)\right]} .
$$

Set $G_{+}^{0}(z)=\lambda_{0} G_{+}^{1}(z)+\left(1-\lambda_{0}\right) G_{+}^{2}(z)$, and $G_{+}^{*}(z)=\frac{a_{+} G_{+}^{0}(z)}{\int_{z_{1}}^{1} G_{+}^{0}(z) d\left[-w_{+}(1-z)\right]}>0$, then

$$
\int_{z_{1}}^{1} G_{+}^{*}(z) d\left[-w_{+}(1-z)\right]=a_{+}
$$

and

$$
\int_{z_{1}}^{1} G_{+}^{*}(z) M_{+}(z) d\left[-w_{+}(1-z)\right]=\frac{a_{+} \int_{z_{1}}^{1} G_{+}^{0}(z) M_{+}(z) d\left[-w_{+}(1-z)\right]}{\int_{z_{1}}^{1} G_{+}^{0}(z) d\left[-w_{+}(1-z)\right]}=a_{+} \frac{y_{+}}{a_{+}}=y_{+},
$$

which shows that $G_{+}^{*}(z)$ is feasible.

Conversely, if a feasible solution, $G_{+}(z)$, exists, it is obvious that

$$
\frac{y_{+}}{a_{+}}=\frac{\int_{z_{1}}^{1} G_{+}(z) M_{+}(z) d\left[-w_{+}(1-z)\right]}{\int_{z_{1}}^{1} G_{+}(z) d\left[-w_{+}(1-z)\right]} \in[\underline{b}, \bar{b}] .
$$

This completes the proof.

Next we give some more explicit conditions for the feasibility of problem (2.11) in the following three propositions. We assume $\frac{y_{+}}{a_{+}} \in(\underline{b}, \bar{b})$ in the following.

\section{Proposition 2.4}

(1) If there exists a $z \in\left(z_{1}, 1\right)$ such that

$$
\int_{z}^{1}\left[M_{+}(x)-\mathbb{E}_{+}^{z_{1}}(\rho)\right] d\left[-w_{+}(1-x)\right]<0,
$$

we have $\underline{b}<\mathbb{E}_{+}^{z_{1}}(\rho)$, and then problem (2.11) has a feasible solution for $\frac{y_{+}}{a_{+}} \in\left(\underline{b}, \mathbb{E}_{+}^{z_{1}}(\rho)\right)$.

(2) Otherwise, if

$$
\int_{z}^{1}\left[M_{+}(x)-\mathbb{E}_{+}^{z_{1}}(\rho)\right] d\left[-w_{+}(1-x)\right] \geq 0
$$

for any $z \in\left(z_{1}, 1\right)$, we have $\underline{b}=\mathbb{E}_{+}^{z_{1}}(\rho)$. Then problem (2.11) has no feasible solution for $\frac{y_{+}}{a_{+}} \in\left(\underline{b}, \mathbb{E}_{+}^{z_{1}}(\rho)\right)$, because $\left(\underline{b}, \mathbb{E}_{+}^{z_{1}}(\rho)\right)$ is empty.

Proof. The proof of this proposition uses a similar argument to that in Theorem 3.1 in Bi et al. (2013), so we omit it here.

\section{Proposition 2.5}

(1) If there exists a $z \in\left(z_{1}, 1\right)$ such that

$$
\int_{z}^{1}\left[M_{+}(x)-\mathbb{E}_{+}^{z_{1}}(\rho)\right] d\left[-w_{+}(1-x)\right]>0,
$$

we have $\mathbb{E}_{+}^{z_{1}}(\rho)<\bar{b}$, and then problem (2.11) has a feasible solution for $\frac{y_{+}}{a_{+}} \in\left(\mathbb{E}_{+}^{z_{1}}(\rho), \bar{b}\right)$. 
(2) Otherwise, if

$$
\int_{z}^{1}\left[M_{+}(x)-\mathbb{E}_{+}^{z_{1}}(\rho)\right] d\left[-w_{+}(1-x)\right] \leq 0
$$

for any $z \in\left(z_{1}, 1\right)$, we have $\mathbb{E}_{+}^{z_{1}}(\rho)=\bar{b}$. Problem (2.11) has no feasible solution for $\frac{y_{+}}{a_{+}} \in\left(\mathbb{E}_{+}^{z_{1}}(\rho), \bar{b}\right)$, because $\left(\mathbb{E}_{+}^{z_{1}}(\rho), \bar{b}\right)$ is empty.

Proposition 2.6 If $\frac{y_{+}}{a_{+}}=\mathbb{E}_{+}^{z_{1}}(\rho)$, there exists a feasible solution for problem (2.11).

Proof. When $\frac{y_{+}}{a_{+}}=\mathbb{E}_{+}^{z_{1}}(\rho)$, one can directly verify that $G_{+}(\cdot)=\frac{a_{+}}{\int_{z_{1}}^{1} w_{+}^{\prime}(1-z) d z}$ is a feasible solution of problem (2.11).

Remark 2.2 In Proposition 2.4(1), we cannot guarantee the existence of a feasible solution for $\frac{y_{+}}{a_{+}} \in\left(\mathbb{E}_{+}^{z_{1}}(\rho), \bar{b}\right)$. Actually, $\mathbb{E}_{+}^{z_{1}}(\rho)$ maybe equal to $\bar{b}$. For example, if

$$
\int_{z}^{1}\left[M_{+}(x)-\mathbb{E}_{+}^{z_{1}}(\rho)\right] d\left[-w_{+}(1-x)\right] \leq 0
$$

for any $z \in\left(z_{1}, 1\right)$, we have $\mathbb{E}_{+}^{z_{1}}(\rho)=\bar{b}$, then $\left(\mathbb{E}_{+}^{z_{1}}(\rho), \bar{b}\right)$ will be empty. The details can be found in Proposition 2.5(2).

Remark 2.3 In Proposition 2.5(1), we cannot guarantee the existence of a feasible solution for $\frac{y_{+}}{a_{+}} \in\left(\underline{b}, \mathbb{E}_{+}^{z_{1}}(\rho)\right)$. Actually, $\mathbb{E}_{+}^{z_{1}}(\rho)$ maybe equal to $\underline{b}$. For example, if

$$
\int_{z}^{1}\left[M_{+}(x)-\mathbb{E}_{+}^{z_{1}}(\rho)\right] d\left[-w_{+}(1-x)\right] \geq 0
$$

for any $z \in\left(z_{1}, 1\right)$, we have $\mathbb{E}_{+}^{z_{1}}(\rho)=\underline{b}$, then $\left(\underline{b}, \mathbb{E}_{+}^{z_{1}}(\rho)\right)$ will be empty. The details can be found in Proposition 2.4(2).

Remark 2.4 From Proposition 2.4 and Proposition 2.5, we can see that, if there exist $\bar{z}, \tilde{z} \in$ $\left(z_{1}, 1\right)$ such that

$$
\int_{\bar{z}}^{1}\left[M_{+}(x)-\mathbb{E}_{+}^{z_{1}}(\rho)\right] d\left[-w_{+}(1-x)\right]>0
$$

and

$$
\int_{\tilde{z}}^{1}\left[M_{+}(x)-\mathbb{E}_{+}^{z_{1}}(\rho)\right] d\left[-w_{+}(1-x)\right]<0,
$$

then we have $\underline{b}<\mathbb{E}_{+}(\rho)<\bar{b}$. So there exists a feasible solution for problem (2.11) for both $\frac{y_{+}}{a_{+}} \in\left(\underline{b}, \mathbb{E}_{+}^{z_{1}}(\rho)\right)$ and $\frac{y_{+}}{a_{+}} \in\left(\mathbb{E}_{+}^{z_{1}}(\rho), \bar{b}\right)$.

\section{Optimal solution for the positive part problem and negative part problem}

The next two sections are devoted to solving for the optimal attainable wealth, which is the focus of the first subproblem and problem (2.5). In this section, we solve the problems of the positive and negative parts in Step 1. In Section 4, we talk about problem (2.13) from Step 2, in which we merged the positive and the negative part problems.

In Subsection 3.1, in order to eliminate the constraint conditions in the positive and negative part problems, we introduce the Lagrange multipliers $\left(\lambda_{+}, \mu_{+}\right),\left(\lambda_{-}, \mu_{-}\right)$into both problems. For the convenience of calculation, we solve an auxiliary problem in Subsection 3.2. In Subsections 3.3-3.4, we study the optimal solution for the positive and negative part problems in four typical but sufficiently general cases of probability distortions. 


\subsection{Problem transformation}

Before considering the positive and negative part problems, we present the following theorem.

Theorem 3.1 Assume that $z_{1}$ and $a_{+}$are fixed.

i) Under the condition of Proposition 2.4(1), the value function of the positive part, problem (2.9), is decreasing for $y_{+} \in\left(a_{+} \underline{b}, a_{+} \mathbb{E}_{+}^{z_{1}}(\rho)\right)$. Under the condition of Proposition 2.5(1), the value function of the positive part, problem (2.9), is increasing for $y_{+} \in$ $\left(a_{+} \mathbb{E}_{+}^{z_{1}}(\rho), a_{+} \bar{b}\right)$.

ii) If the feasible solution exists for the negative part, problem (2.10), the value function of problem (2.10) is decreasing for $y_{+}-y_{0}<a_{+} \mathbb{E}_{-}^{z_{1}}(\rho)$, and increasing for $y_{+}-y_{0}>a_{+} \mathbb{E}_{-}^{z_{1}}(\rho)$.

Proof. If the feasible solution for problem (2.9) exists (see the results in Subsection 2.5), then the existence of an optimal solution for problem (2.9) is easily apparent. We show the optimal solution in Subsections 3.3-3.4.

i) Set $a_{+} \underline{b}<\bar{y}_{+}<\hat{y}_{+}<a_{+} \mathbb{E}_{+}^{z_{1}}(\rho)$, we shall prove that $v_{+}\left(z_{1}, a_{+}, \hat{y}_{+}\right)<v_{+}\left(z_{1}, a_{+}, \bar{y}_{+}\right)$, where $v_{+}\left(z_{1}, a_{+}, \hat{y}_{+}\right)$and $v_{+}\left(z_{1}, a_{+}, \bar{y}_{+}\right)$are the optimal values of problem $(2.9)$ that correspond to $\hat{y}_{+}$and $\bar{y}_{+}$respectively. Let $\bar{G}_{+}(\cdot)$ denote the optimal solution for (2.9) when the parameters are $\left(z_{1}, a_{+}, \bar{y}_{+}\right)$. Recall that the optimal solution $\bar{G}_{+}(\cdot) \in \mathbb{G}_{+}$for $(2.9)$ is the positive part of the optimal quantile function, $G^{*}(\cdot)$, for problem (2.8). After solving problem (2.8) with the optimal solution $G^{*}(\cdot)$, the optimal solution (i.e., the optimal terminal wealth) for $(2.7)$ can be recovered by $Y^{*}=G^{*}\left(1-F_{\rho}(\rho)\right)$. Then, the optimal portfolio can be found by Proposition 2.1.

Construct

$$
\hat{G}_{+}(\cdot):=\frac{\hat{y}_{+}-a_{+} \mathbb{E}_{+}^{z_{1}}(\rho)}{\bar{y}_{+}-a_{+} \mathbb{E}_{+}^{z_{1}}(\rho)}\left[\bar{G}_{+}(\cdot)-\frac{a_{+}}{\int_{z_{1}}^{1} w_{+}^{\prime}(1-z) d z}\right]+\frac{a_{+}}{\int_{z_{1}}^{1} w_{+}^{\prime}(1-z) d z} .
$$

It is easy to verify that $\hat{G}_{+}(\cdot)$ satisfies all three of the conditions in problem $(2.9)$ when the parameters are $\left(z_{1}, a_{+}, \hat{y}_{+}\right)$. Because it satisfies those conditions, $\hat{G}_{+}(\cdot)$ is a feasible solution for $(2.9)$ when the parameters are $\left(z_{1}, a_{+}, \hat{y}_{+}\right)$and $v_{+}\left(z_{1}, a_{+}, \hat{y}_{+}\right)<\widetilde{V}\left(\hat{G}_{+}(\cdot)\right)<$ $\widetilde{V}\left(\bar{G}_{+}(\cdot)\right)=v_{+}\left(z_{1}, a_{+}, \bar{y}_{+}\right)$, where $\widetilde{V}(\cdot)$ is the behavioral variance. Thus, we get our result. The result for $y_{+} \in\left(a_{+} \mathbb{E}_{+}^{z_{1}}(\rho), a_{+} \bar{b}\right)$ can be similarly derived.

ii) Using a similar analysis as to that used in i), we can solve for ii).

From the above theorem, we know that for fixed $z_{1}$ and $a_{+}$, if $y_{+} \leq \min \left\{a_{+} \mathbb{E}_{+}^{z_{1}}(\rho), y_{0}+\right.$ $\left.a_{+} \mathbb{E}_{-}^{z_{1}}(\rho)\right\}$, then both $v_{+}\left(z_{1}, a_{+}, y_{+}\right)$and $v_{-}\left(z_{1}, a_{+}, y_{+}\right)$are decreasing for $y_{+}$, and the optimal value for problem (2.13) will be attained at $y_{+}=\min \left\{a_{+} \mathbb{E}_{+}^{z_{1}}(\rho), y_{0}+a_{+} \mathbb{E}_{-}^{z_{1}}(\rho)\right\}$. On the other hand, if $y_{+} \geq \max \left\{a_{+} \mathbb{E}_{+}^{z_{1}}(\rho), y_{0}+a_{+} \mathbb{E}_{-}^{z_{1}}(\rho)\right\}$, then both $v_{+}\left(z_{1}, a_{+}, y_{+}\right)$and $v_{-}\left(z_{1}, a_{+}, y_{+}\right)$ are increasing for $y_{+}$, and the optimal value for problem (2.13) will be attained at $y_{+}=$ $\max \left\{a_{+} \mathbb{E}_{+}^{z_{1}}(\rho), y_{0}+a_{+} \mathbb{E}_{-}^{z_{1}}(\rho)\right\}$. So next, we only need to consider the parameter interval

$$
\min \left\{a_{+} \mathbb{E}_{+}^{z_{1}}(\rho), y_{0}+a_{+} \mathbb{E}_{-}^{z_{1}}(\rho)\right\} \leq y_{+} \leq \max \left\{a_{+} \mathbb{E}_{+}^{z_{1}}(\rho), y_{0}+a_{+} \mathbb{E}_{-}^{z_{1}}(\rho)\right\} .
$$

Note that the positive part, problem (2.11), and the negative part, problem (2.12), are convex in $G(\cdot)$, so we can remove the constraints of both problems by introducing Lagrange multipliers $\left(\lambda_{+}, \mu_{+}\right)$and $\left(\lambda_{-}, \mu_{-}\right)$, and then consider the following problems, 


$$
\begin{aligned}
\min _{G_{+} \in \mathbb{G}_{+}} & \int_{z_{1}}^{1} G_{+}(z)^{2} w_{+}^{\prime}(1-z) d z-2 \lambda_{+}\left[\int_{z_{1}}^{1} G_{+}(z) w_{+}^{\prime}(1-z) d z-a_{+}\right] \\
+ & 2 \mu_{+}\left[\int_{z_{1}}^{1} G_{+}(z) F_{\rho}^{-1}(1-z) d z-y_{+}\right] \\
=\min _{G_{+} \in \mathbb{G}_{+}} & \int_{z_{1}}^{1}\left[G_{+}(z)-\left(\lambda_{+}-\mu_{+} M_{+}(z)\right)\right]^{2} w_{+}^{\prime}(1-z) d z \\
& -\int_{z_{1}}^{1}\left(\lambda_{+}-\mu_{+} M_{+}(z)\right)^{2} w_{+}^{\prime}(1-z) d z+2 \lambda_{+} a_{+}-2 \mu_{+} y_{+},
\end{aligned}
$$

and

$$
\begin{aligned}
\min _{G_{-} \in \mathbb{G}_{-}} & \int_{0}^{z_{1}} G_{-}(z)^{2} w_{-}^{\prime}(z) d z-2 \lambda_{-}\left[\int_{0}^{z_{1}} G_{-}(z) w_{-}^{\prime}(z) d z-a_{+}\right] \\
& +2 \mu_{-}\left[\int_{0}^{z_{1}} G_{-}(z) F_{\rho}^{-1}(1-z) d z-y_{+}+y_{0}\right] \\
=\min _{G_{-} \in \mathbb{G}_{-}} & \int_{0}^{z_{1}}\left[G_{-}(z)-\left(\lambda_{-}-\mu_{-} M_{-}(z)\right)\right]^{2} w_{-}^{\prime}(z) d z-\int_{0}^{z_{1}}\left(\lambda_{-}-\mu_{-} M_{-}(z)\right)^{2} w_{-}^{\prime}(z) d z \\
& +2 \lambda_{-} a_{+}-2 \mu_{-} y_{+}+2 \mu_{-} y_{0} .
\end{aligned}
$$

After solving these two problems, we determine the Lagrange multipliers using the original constraints.

Note that if $\mu_{+}=0$, the optimal solution for problem (3.1) is $G_{+}^{*}(z)=\left(\lambda_{+}\right)^{+}$. Insert it into the constraints in problem (2.11), we know that the constraint admits no solution except $\frac{y_{+}}{a_{+}}=$ $\mathbb{E}_{+}^{z_{1}}(\rho)$. When $\frac{y_{+}}{a_{+}}=\mathbb{E}_{+}^{z_{1}}(\rho)$, the optimal solution for problem $(2.11)$ is $G_{+}^{*}(z)=\frac{a_{+}}{\int_{z_{1}}^{1} w_{+}^{\prime}(1-z) d z}$. Moreover, if $\mu_{-}=0$, the optimal solution for problem $(3.2)$ is $G_{-}^{*}(z)=\left(\lambda_{-}\right)^{+}$. Insert it into the constraints in (2.12), we know that the constraint admits no solution except $\frac{y_{+}-y_{0}}{a_{+}}=\mathbb{E}_{-}^{z_{1}}(\rho)$. When $\frac{y_{+}-y_{0}}{a_{+}}=\mathbb{E}_{-}^{z_{1}}(\rho)$, the optimal solution for problem $(2.12)$ is $G_{-}^{*}(z)=\frac{a_{+}}{\int_{0}^{z_{1}} w_{-}^{\prime}(z) d z}$. Thus next we consider the case $\frac{y_{+}}{a_{+}} \neq \mathbb{E}_{+}^{z_{1}}(\rho)$ and $\frac{y_{+}-y_{0}}{a_{+}} \neq \mathbb{E}_{-}^{z_{1}}(\rho)$, then $\mu_{+} \neq 0$ and $\mu_{-} \neq 0$.

Using Theorem 3.1, we know that if $a_{+} \mathbb{E}_{+}^{z_{1}}(\rho)<y_{0}+a_{+} \mathbb{E}_{-}^{z_{1}}(\rho)$, and $y_{+} \in\left[a_{+} \mathbb{E}_{+}^{z_{1}}(\rho), y_{0}+\right.$ $\left.a_{+} \mathbb{E}_{-}^{z_{1}}(\rho)\right]$, we have $\mu_{+} \leq 0, \mu_{-} \geq 0$. Conversely, if $y_{0}+a_{+} \mathbb{E}_{-}^{z_{1}}(\rho)<a_{+} \mathbb{E}_{+}^{z_{1}}(\rho)$ and $y_{+} \in$ $\left[y_{0}+a_{+} \mathbb{E}_{-}^{z_{1}}(\rho), a_{+} \mathbb{E}_{+}^{z_{1}}(\rho)\right]$, we have $\mu_{+} \geq 0, \mu_{-} \leq 0$.

Analogous to the cases considered in Bi et al. (2013), we consider the following four typical cases of $M_{ \pm}(z)$ which has two monotone pieces, where $0 \leq a<b \leq 1$.

- Case 1: There exists a $z_{0} \in[a, b]$, such that $M_{ \pm}(z)$ is strictly increasing across $\left(a, z_{0}\right)$, and strictly decreasing across $\left(z_{0}, b\right)$, and $M_{ \pm}(a)<M_{ \pm}(b)$. (See Figure 1.)

- Case 2: There exists a $z_{0} \in[a, b]$, such that $M_{ \pm}(z)$ is strictly increasing across $\left(a, z_{0}\right)$, and strictly decreasing across $\left(z_{0}, b\right)$, and $M_{ \pm}(a) \geq M_{ \pm}(b)$. (See Figure 2.)

- Case 3: There exists a $z_{0} \in[a, b]$, such that $M_{ \pm}(z)$ is strictly decreasing across $\left(a, z_{0}\right)$, and strictly increasing across $\left(z_{0}, b\right)$, and $M_{ \pm}(a) \geq M_{ \pm}(b)$. (See Figure 3.)

- Case 4: There exists a $z_{0} \in[a, b]$, such that $M_{ \pm}(z)$ is strictly decreasing across $\left(a, z_{0}\right)$, and strictly increasing across $\left(z_{0}, b\right)$, and $M_{ \pm}(a)<M_{ \pm}(b)$. (See Figure 4.) 
Remark 3.1 Note that the case where $M_{ \pm}(z)$ has a finite number of monotonic pieces only incurs notational complexity in the approach below, but manifests no essential difference. So, if the optimal solutions for the positive and negative part problems are derived with $M_{ \pm}(z)$ in the above four cases, we can obtain an optimal solution for the more general case when $M_{ \pm}(z)$ has a finite number of monotonic pieces using a similar analysis.

Based on Propositions 2.4 and 2.5, we first consider the feasibility of the positive part problem under the above four cases of $M_{ \pm}(z)$.

\section{Proposition 3.1}

i) Assume $\frac{y_{+}}{a_{+}} \in\left(\underline{b}, \mathbb{E}_{+}^{z_{1}}(\rho)\right)$. There is no feasible solution for problem (2.11) in Case 1 with $M_{+}\left(z_{1}\right)<\mathbb{E}_{+}^{z_{1}}(\rho)<M_{+}(1)$ and Case 4 with $M_{+}\left(z_{1}\right)<\mathbb{E}_{+}^{z_{1}}(\rho)<M_{+}(1)$. Otherwise, there exists a feasible solution for problem (2.11).

ii) Assume $\frac{y_{+}}{a_{+}}=\mathbb{E}_{+}^{z_{1}}(\rho)$. There exists at least one feasible solution $G_{+}(\cdot)=\frac{a_{+}}{\int_{z_{1}}^{1} w_{+}^{\prime}(1-z) d z}$ for problem (2.11), no matter which case $M_{+}(z)$ is included in.

iii) Assume $\frac{y_{+}}{a_{+}} \in\left(\mathbb{E}_{+}^{z_{1}}(\rho), \bar{b}\right)$. There is no feasible solution for problem (2.11) in Case 2 with $M_{+}(1)<\mathbb{E}_{+}^{z_{1}}(\rho)<M_{+}\left(z_{1}\right)$ and Case 3 with $M_{+}(1)<\mathbb{E}_{+}^{z_{1}}(\rho)<M_{+}\left(z_{1}\right)$. Otherwise, there exists a feasible solution for problem (2.11).

\section{Proof.}

i) Define $g(z)=\int_{z}^{1}\left[M_{+}(x)-\mathbb{E}_{+}^{z_{1}}(\rho)\right] d\left[-w_{+}(1-x)\right]$, where $z \in\left[z_{1}, 1\right]$. It is easy to see that $g\left(z_{1}\right)=g(1)=0$. Because $M_{+}\left(z_{1}\right)<\mathbb{E}_{+}^{z_{1}}(\rho)<M_{+}(1)$ and because the shape of $M_{+}(z)$, we have $g(z)$ is increasing in $\left(z_{1}, M_{+}^{-1}\left(\mathbb{E}_{+}^{z_{1}}(\rho)\right)\right)$ and decreasing in $\left(M_{+}^{-1}\left(\mathbb{E}_{+}^{z_{1}}(\rho)\right), 1\right)$. Thus $g(z) \geq 0$ for $z \in\left[z_{1}, 1\right]$. According to Proposition 2.4(2), there exists no feasible solution for problem (2.11).

In other cases, it is easy to verify that the feasible condition in Proposition 2.4(1) is satisfied, thereby showing that there exists a feasible solution for problem (2.11).

ii) When $\frac{y_{+}}{a_{+}}=\mathbb{E}_{+}^{z_{1}}(\rho)$, one can directly verify that $G_{+}(\cdot)=\frac{a_{+}}{\int_{z_{1}}^{1} w_{+}^{\prime}(1-z) d z}$ is a feasible solution for problem (2.11).

iii) Using Proposition 2.5, the proof of iii) is similar to that of i).

We can derive the feasibility of the negative part problem under the four cases, in the same process that we determined the feasibility of the positive part problem. Because of this, we only show the result in the following proposition and omit the proof for the negative part problem.

Proposition 3.2 Assume that $y_{+}, a_{+}$satisfy the basic feasibility condition for the negative part problem, which is similar to that for the positive part problem in Proposition 2.3.

i) Assume $\frac{y_{+}-y_{0}}{a_{+}}>\mathbb{E}_{-}^{z_{1}}(\rho)$. There is no feasible solution for problem (2.12) in Case 1 with $M_{-}(0)<\mathbb{E}_{-}^{z_{1}}(\rho)<M_{-}\left(z_{1}\right)$ and Case 4 with $M_{-}(0)<\mathbb{E}_{-}^{z_{1}}(\rho)<M_{-}\left(z_{1}\right)$. Otherwise, there exists a feasible solution for problem (2.12).

ii) Assume $\frac{y_{+}-y_{0}}{a_{+}}=\mathbb{E}_{-}^{z_{1}}(\rho)$. There exists at least one feasible solution $G_{-}(\cdot)=\frac{a_{+}}{\int_{0}^{z_{1}} w_{-}^{\prime}(z) d z}$ for problem (2.12), no matter which case $M_{-}(z)$ is included in. 
iii) Assume $\frac{y_{+}-y_{0}}{a_{+}}<\mathbb{E}_{-}^{z_{1}}(\rho)$. There is no feasible solution for problem (2.12) in Case 2 with $M_{-}\left(z_{1}\right)<\mathbb{E}_{-}^{z_{1}}(\rho)<M_{-}(0)$ and Case 3 with $M_{-}\left(z_{1}\right)<\mathbb{E}_{-}^{z_{1}}(\rho)<M_{-}(0)$. Otherwise, there exists a feasible solution for problem (2.12).

Remark 3.2 The intuitive explanations of the above two propositions are: The feasibility reflects the coordination among the probability distortions and the market (represented by $\rho$ ) in a mathematical way. In other words, the feasibility condition model requires some consistency between the probability distortion and the market. In Propositions 3.1 and 3.2, we show that some forms of $M_{ \pm}(z)$ do not satisfy the feasibility condition, therefore also showing that there exists no feasible solution for problems (2.11) and (2.12).

\subsection{The auxiliary problem}

For the convenience of calculation, we shall solve an auxiliary problem in this subsection. Consider problem

$$
\min _{G \in \mathbb{G}} \int_{a}^{b}[G(z)-f(z)]^{2} w^{\prime}(1-z) d z,
$$

where $w^{\prime}(1-z)>0$, and represents $w_{+}^{\prime}(1-z)$ in the positive part problem, or $w_{-}^{\prime}(z)$ in the negative part problem. $f(z)$ represents $\lambda_{+}-\mu_{+} M_{+}(z)$ in the positive part problem, or $\lambda_{-}-\mu_{-} M_{-}(z)$ in the negative part problem. Notice that the optimal solution for problem (3.3) depends mainly on the shape of $f(z)$. First, to get started, we give the following lemma.

Lemma 3.1 Consider problem (3.3).

i) If $f(z)$ is in Case 1, the optimal solution for problem (3.3) takes the form $G(z)=$ $f(z) \mathbf{1}_{a<z \leq z_{2}}+f\left(z_{2}\right) 1_{z_{2}<z<b}$, where $z_{2} \in\left[\bar{z}_{1}, z_{0}\right]$ is to be determined and $\bar{z}_{1} \in\left(a, z_{0}\right)$ is the part of the solution that satisfied $f\left(\bar{z}_{1}\right)=f(b)$.

ii) If $f(z)$ is in Case 2, the optimal solution for problem (3.3) has two possible forms, one is $G(z) \equiv K \in[f(b), f(a)]$, where $z \in[a, b]$, and the other is $G(z)=f(z) \mathbf{1}_{a<z \leq z_{2}}+$ $f\left(z_{2}\right) \boldsymbol{1}_{z_{2}<z \leq b}$, where $z_{2} \in\left[a, z_{0}\right]$ is to be determined.

iii) If $f(z)$ is in Case 3, the optimal solution for problem (3.3) has two possible forms, one is $G(z) \equiv K \in[f(b), f(a)]$, where $z \in[a, b]$, and the other is $G(z)=f\left(z_{2}\right) \mathbf{1}_{a<z \leq z_{2}}+$ $f(z) \mathbf{1}_{z_{2}<z \leq b}$, where $z_{2} \in\left[z_{0}, b\right]$ is to be determined.

iv) If $f(z)$ is in Case 4, the optimal solution for problem (3.3) has the form $G(z)=f\left(z_{2}\right) 1_{a<z \leq z_{2}}+$ $f(z) \boldsymbol{1}_{z_{2}<z \leq b}$, where $z_{2} \in\left[z_{0}, \bar{z}_{1}\right]$ is to be determined and $\bar{z}_{1} \in\left(z_{0}, b\right)$ is the value at which $f\left(\bar{z}_{1}\right)=f(a)$.

Proof. The proof of this lemma is similar to that of Lemma 3.9 and Lemma 3.10 in Bi et al. (2013), so we omit it. We just show the result of this lemma in Figures 1-4.

On account of Theorem 3.1, in order to solve the positive part, problem (2.11), and the negative part, problem (2.12), we only need to consider the following two situations. First, if $a_{+} \mathbb{E}_{+}^{z_{1}}(\rho)<y_{0}+a_{+} \mathbb{E}_{-}^{z_{1}}(\rho)$, and then $y_{+} \in\left(a_{+} \mathbb{E}_{+}^{z_{1}}(\rho), y_{0}+a_{+} \mathbb{E}_{-}^{z_{1}}(\rho)\right)$. Second, if $y_{0}+a_{+} \mathbb{E}_{-}^{z_{1}}(\rho)<$ $a_{+} \mathbb{E}_{+}^{z_{1}}(\rho)$, and then $y_{+} \in\left(y_{0}+a_{+} \mathbb{E}_{-}^{z_{1}}(\rho), a_{+} \mathbb{E}_{+}^{z_{1}}(\rho)\right)$. In Subsection 3.3, we give the optimal solutions for the positive part, problem (2.11), and the negative part, problem (2.12), when $a_{+} \mathbb{E}_{+}^{z_{1}}(\rho)<y_{0}+a_{+} \mathbb{E}_{-}^{z_{1}}(\rho)$. In Subsection 3.4, we give the optimal solutions for the positive part, problem (2.11), and the negative part, problem (2.12), when $y_{0}+a_{+} \mathbb{E}_{-}^{z_{1}}(\rho)<a_{+} \mathbb{E}_{+}^{z_{1}}(\rho)$. 


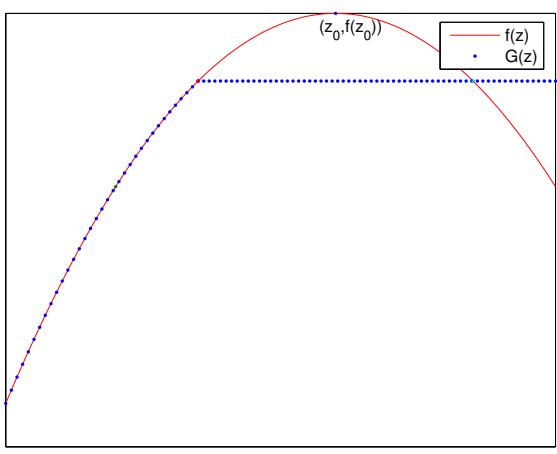

Fig. 1. $f(z)$ and $G(z)$ in Lemma 3.1 i)

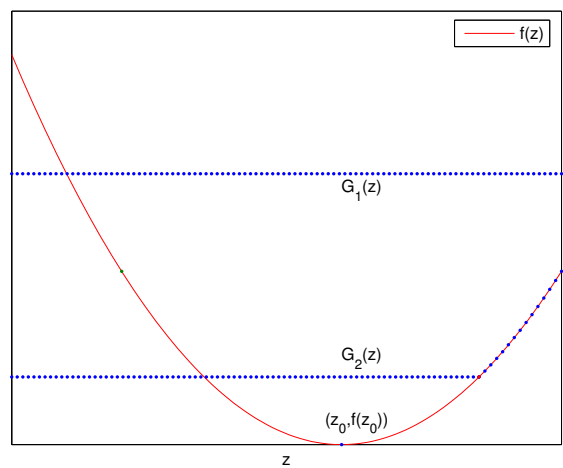

Fig. 3. $f(z)$ and $G(z)$ in Lemma 3.1 iii)

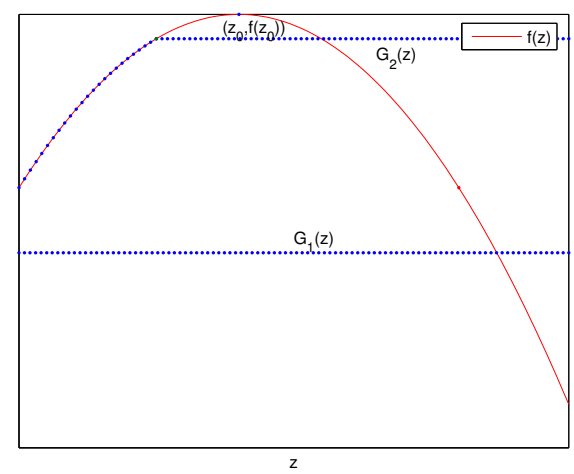

Fig. 2. $f(z)$ and $G(z)$ in Lemma 3.1 ii)

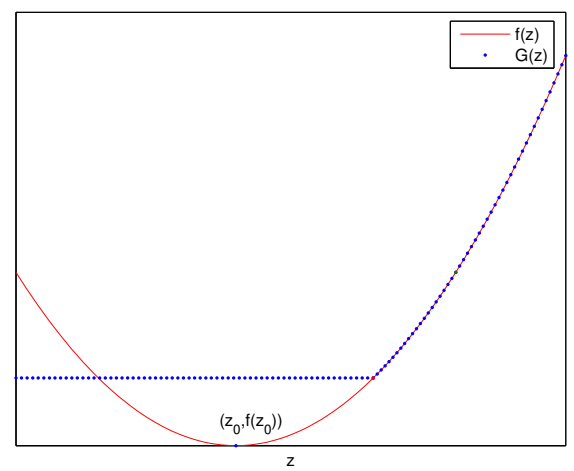

Fig. 4. $f(z)$ and $G(z)$ in Lemma 3.1 iv) 


\subsection{The optimal solution for problems (2.11) and (2.12) if $a_{+} \mathbb{E}_{+}^{z_{1}}(\rho) \leq y_{0}+$ $a_{+} \mathbb{E}_{-}^{z_{1}}(\rho)$}

In this subsection we solve the positive part, problem (2.11), and the negative part, problem (2.12), under the situation where $a_{+} \mathbb{E}_{+}^{z_{1}}(\rho) \leq y_{0}+a_{+} \mathbb{E}_{-}^{z_{1}}(\rho)$. Under this situation, we know from Theorem 3.1 that we only need to consider the parameter interval $y_{+} \in\left[a_{+} \mathbb{E}_{+}^{z_{1}}(\rho), y_{0}+a_{+} \mathbb{E}_{-}^{z_{1}}(\rho)\right]$. Furthermore, we know from Theorem 3.1 that $\mu_{+} \leq 0$ and $\mu_{-} \geq 0$ if $y_{+} \in\left[a_{+} \mathbb{E}_{+}^{z_{1}}(\rho), y_{0}+\right.$ $\left.a_{+} \mathbb{E}_{-}^{z_{1}}(\rho)\right]$. We solve the positive part problem and the negative part problem in Subsection 3.3.1 and Subsection 3.3.2, respectively.

\subsubsection{The optimal solution for the positive part problem (2.11)}

We assume $M_{+}(z)$ has two monotonic intervals, $\left(z_{1}, z_{0+}\right)$ and $\left(z_{0+}, 1\right)$. Thus, $M_{+}(z)$ is in one of the above four cases with $z_{0}=z_{0+}, a=z_{1}$ and $b=1$. From Proposition 3.1, we know that if $M_{+}(z)$ is in Case 2 with $\mathbb{E}_{+}^{z_{1}}(\rho) \in\left[M_{+}(1), M_{+}\left(z_{1}\right)\right)$ or Case 3 with $\mathbb{E}_{+}^{z_{1}}(\rho) \in\left[M_{+}(1), M_{+}\left(z_{1}\right)\right)$, there exists no feasible solution for problem (2.11). So next, we consider the optimal solutions for problem (3.1) under other cases.

Consider the following auxiliary problem

$$
\min _{G_{+} \in \mathbb{G}} \int_{z_{1}}^{1}\left[G_{+}(z)-\left(\lambda_{+}-\mu_{+} M_{+}(z)\right)\right]^{2} w_{+}^{\prime}(1-z) d z,
$$

which is introduced in Subsection 3.2. The solution to problem (3.4) is summarized in the following lemma.

Lemma $3.2 \quad$ i) Assume $M_{+}(z)$ is from Case 1 , or from Case 2 with $\mathbb{E}_{+}^{z_{1}}(\rho)>M_{+}\left(z_{1}\right)$, then the optimal solution for (3.4) is

$$
G_{+}^{*}(z)=\left[\lambda_{+}-\mu_{+} M_{+}(z)\right] \mathbf{1}_{z_{1}<z \leq z_{+}^{*}}+\left[\lambda_{+}-\mu_{+} M_{+}\left(z_{+}^{*}\right)\right] \mathbf{1}_{z_{+}^{*}<z \leq 1},
$$

where $z_{+}^{*}$ is determined by

$$
\int_{z_{+}^{*}}^{1}\left[M_{+}\left(z_{+}^{*}\right)-M_{+}(z)\right] w_{+}^{\prime}(1-z) d z=0 .
$$

In Case 1 , we have $z_{+}^{*} \in\left[\bar{z}_{0+}, z_{0+}\right]$ and $\bar{z}_{0+} \in\left(z_{1}, z_{0+}\right)$ is the value at which $M_{+}\left(\bar{z}_{0+}\right)=$ $M_{+}(1)$. In Case 2 with $\mathbb{E}_{+}^{z_{1}}(\rho)>M_{+}\left(z_{1}\right)$, we have $z_{+}^{*} \in\left[z_{1}, z_{0+}\right]$.

ii) Assume $M_{+}(z)$ is from Case 3 with $\mathbb{E}_{+}^{z_{1}}(\rho)<M_{+}(1)$, or from Case 4, then the optimal solution for (3.4) is

$$
G_{+}^{*}(z)=\left[\lambda_{+}-\mu_{+} M_{+}\left(z_{+}^{*}\right)\right] \mathbf{1}_{z_{1}<z \leq z_{+}^{*}}+\left[\lambda_{+}-\mu_{+} M_{+}(z)\right] \mathbf{1}_{z_{+}^{*}<z \leq 1},
$$

where $z_{+}^{*}$ is determined by

$$
\int_{z_{1}}^{z_{+}^{*}}\left[M_{+}\left(z_{+}^{*}\right)-M_{+}(z)\right] w_{+}^{\prime}(1-z) d z=0 .
$$

In Case 3 when $\mathbb{E}_{+}^{z_{1}}(\rho)<M_{+}(1)$, then $z_{+}^{*} \in\left[z_{0+}, 1\right]$. In Case 4, we have $z_{+}^{*} \in\left[z_{0+}, \bar{z}_{0+}\right]$, and $\bar{z}_{0+} \in\left(z_{0+}, 1\right)$ is the value at which $M_{+}\left(\bar{z}_{0+}\right)=M_{+}\left(z_{1}\right)$. 
Proof. The proof is given in Appendix A.

In the following theorem, we use Lemma 3.2 to solve for the optimal solution for problem (3.1), in which we involve the Lagrange multipliers, $\left(\lambda_{+}, \mu_{+}\right)$.

Theorem 3.2 i) Assume $M_{+}(z)$ is from Case 1 , or from Case 2 with $\mathbb{E}_{+}^{z_{1}}(\rho)>M_{+}\left(z_{1}\right)$, then the optimal solution for (3.1) is

$$
G_{+}^{*}(z)=\left[\lambda_{+}-\mu_{+} M_{+}(z)\right]^{+} \mathbf{1}_{z_{1}<z \leq z_{+}^{*}}+\left[\lambda_{+}-\mu_{+} M_{+}\left(z_{+}^{*}\right)\right]^{+} \mathbf{1}_{z_{+}^{*}<z \leq 1},
$$

where $z_{+}^{*}$ is determined by (3.5).

ii) Assume $M_{+}(z)$ is from Case 3 with $\mathbb{E}_{+}^{z_{1}}(\rho)<M_{+}(1)$, or from Case 4 , then the optimal solution for (3.1) is

$$
G_{+}^{*}(z)=\left[\lambda_{+}-\mu_{+} M_{+}\left(z_{+}^{*}\right)\right]^{+} \mathbf{1}_{z_{1}<z \leq z_{+}^{*}}+\left[\lambda_{+}-\mu_{+} M_{+}(z)\right]^{+} \mathbf{1}_{z_{+}^{*}<z \leq 1},
$$

where $z_{+}^{*}$ is determined by (3.6).

\section{Proof.}

i) The difference between problems (3.4) and (3.1) is the constraint in problem (3.1) that $G_{+}(z) \geq 0$. Once we solve for Case 1 , the results based around the other cases can be obtained in the same way. If $M_{+}\left(z_{1}\right) \geq 0$, then

$$
\begin{aligned}
& {\left[\lambda_{+}-\mu_{+} M_{+}(z)\right] \mathbf{1}_{z_{1}<z \leq z_{+}^{*}}+\left[\lambda_{+}-\mu_{+} M_{+}\left(z_{+}^{*}\right)\right] \mathbf{1}_{z_{+}^{*}<z \leq 1} } \\
= & {\left[\lambda_{+}-\mu_{+} M_{+}(z)\right]^{+} \mathbf{1}_{z_{1}<z \leq z_{+}^{*}}+\left[\lambda_{+}-\mu_{+} M_{+}\left(z_{+}^{*}\right)\right]^{+} \mathbf{1}_{z_{+}^{*}<z \leq 1} }
\end{aligned}
$$

is the optimal solution for problem (3.1). If $M_{+}\left(z_{1}\right)<0$ and $M_{+}\left(z_{0+}\right)>0$, then the optimal solution for problem (3.1) takes the form

$$
G_{+}(z)=0 \times \mathbf{1}_{z_{1}<z \leq M_{+}^{-1}\left(\frac{\lambda_{+}}{\mu_{+}}\right)}+\left[\lambda_{+}-\mu_{+} M_{+}(z)\right] \mathbf{1}_{M_{+}^{-1}\left(\frac{\lambda_{+}}{\mu_{+}}\right)<z \leq \bar{z}}+\left[\lambda_{+}-\mu_{+} M_{+}(\bar{z})\right] \mathbf{1}_{\bar{z}<z \leq 1},
$$

where $\bar{z}$ is to be determined. Inserting this into problem (3.1), after a similar calculation from Lemma 3.2, we reach the conclusion that

$$
\bar{z}=\left\{\begin{array}{l}
M_{+}^{-1}\left(\frac{\lambda_{+}}{\mu_{+}}\right), \lambda_{+}-\mu_{+} M_{+}\left(z_{+}^{*}\right)<0 \\
z_{+}^{*}, \lambda_{+}-\mu_{+} M_{+}\left(z_{+}^{*}\right) \geq 0
\end{array}\right.
$$

And the optimal solution for (3.1) is

$$
G_{+}^{*}(z)=\left[\lambda_{+}-\mu_{+} M_{+}(z)\right]^{+} \mathbf{1}_{z_{1}<z \leq z_{+}^{*}}+\left[\lambda_{+}-\mu_{+} M_{+}\left(z_{+}^{*}\right)\right]^{+} \mathbf{1}_{z_{+}^{*}<z \leq 1} .
$$

If $M_{+}\left(z_{1}\right)<0$ and $M_{+}\left(z_{0+}\right) \leq 0$, the optimal solution for problem $(3.1)$ is $G_{+}^{*}(z)=0$, which is also equal to

$$
G_{+}^{*}(z)=\left[\lambda_{+}-\mu_{+} M_{+}(z)\right]^{+} \mathbf{1}_{z_{1}<z \leq z_{+}^{*}}+\left[\lambda_{+}-\mu_{+} M_{+}\left(z_{+}^{*}\right)\right]^{+} \mathbf{1}_{z_{+}^{*}<z \leq 1} .
$$

Once all the situations are summarized, we can formulate our conclusion.

ii) The proof of $i$ ) is similar to that from $i$ ). 
We have shown the optimal solution for (3.1) in Theorem 3.2. In order to get the optimal solution for problem (2.11), we need to determine the Lagrange multipliers, $\left(\lambda_{+}, \mu_{+}\right)$. But it is not easy to get the explicit form of $\left(\lambda_{+}, \mu_{+}\right)$, so we only show the existence and uniqueness of $\left(\lambda_{+}, \mu_{+}\right)$according to the following proposition.

Proposition 3.3 Let $N(\cdot):[0,1] \rightarrow \mathbb{R}^{+}$be a deterministic function. Then equations

$$
\left\{\begin{array}{l}
\mathbb{E}\left[(\lambda-\mu N(Z))^{+}\right]=a_{+} \\
\mathbb{E}\left[(\lambda-\mu N(Z))^{+} N(Z)\right]=y_{+}
\end{array}\right.
$$

have a unique solution $(\lambda, \mu)$ with $\lambda>0, \mu>0$ for any $y_{+}>0, a_{+}>0$, satisfying $\inf \{x$ : $P(N(Z)<x)>0\}<\frac{y_{+}}{a_{+}}<\mathbb{E}[N(Z)]$, and with $\mu<0$ for any $y_{+}>0$, $a_{+}>0$, satisfying $\mathbb{E}[N(Z)]<\frac{y_{+}}{a_{+}}<\sup \{x: P(N(Z)<x)>0\}$. If $\frac{y_{+}}{a_{+}}=\mathbb{E}[N(Z)]$, then the unique solution is $\lambda=a_{+}, \mu=0$.

Proof. The proof of this lemma is similar to that in Bielecki et al. (2005) (Theorem 5.1). So we omit the details of the proof here.

To solve problem (2.11), we only consider two cases: when $M_{+}(z)$ is from Case 3 with $\mathbb{E}_{+}^{z_{1}}(\rho)<M_{+}(1)$, or when it is from Case 4. The conclusion, for all other cases, can be derived similarly, thus we omit those derivations here. Assuming $M_{+}(z)$ is from Case 3 with $\mathbb{E}_{+}^{z_{1}}(\rho)<$ $M_{+}(1)$, or from Case 4, we insert the optimal solution from Theorem 3.2 into the two constraints from (2.11), which gives us

$$
\left\{\begin{array}{l}
\int_{z_{1}}^{z_{+}^{*}}\left[\lambda_{+}-\mu_{+} M_{+}\left(z_{+}^{*}\right)\right]^{+} w_{+}^{\prime}(1-z) d z+\int_{z_{+}^{*}}^{1}\left[\lambda_{+}-\mu_{+} M_{+}(z)\right]^{+} w_{+}^{\prime}(1-z) d z=a_{+} \\
\int_{z_{1}}^{z_{+}^{*}}\left[\lambda_{+}-\mu_{+} M_{+}\left(z_{+}^{*}\right)\right]^{+} F_{\rho}^{-1}(1-z) d z+\int_{z_{+}^{*}}^{1}\left[\lambda_{+}-\mu_{+} M_{+}(z)\right]^{+} F_{\rho}^{-1}(1-z) d z=y_{+} .
\end{array}\right.
$$

Using Proposition 3.3 and (3.6), we know that (3.7) has the unique solution $\mu_{+}\left(z_{1}, a_{+}, y_{+}\right)<0$ and $\lambda_{+}\left(z_{1}, a_{+}, y_{+}\right)$. Thus, we are able to get the optimal solution for problem (2.11), which is shown in Theorem 3.2.

Up to now, we have gotten the optimal solution for the positive part problem (2.11) when $a_{+} \mathbb{E}_{+}^{z_{1}}(\rho)<y_{0}+a_{+} \mathbb{E}_{-}^{z_{1}}(\rho)$. In Subsection 3.3.2, we solve for the negative part problem $(2.12)$ under the same situation.

\subsubsection{The optimal solution for the negative part problem (2.12)}

From Proposition 3.2, we know that if $M_{-}(z)$ is from Case 2 with $\mathbb{E}_{-}^{z_{1}}(\rho) \in\left(M_{-}\left(z_{1}\right), M_{-}(0)\right.$ ] or Case 3 with $\mathbb{E}_{-}^{z_{1}}(\rho) \in\left[M_{-}\left(z_{1}\right), M_{-}(0)\right)$, then there exists no feasible solution for problem (2.12). Because of this, we next consider the optimal solution for problem (3.2) in the relation to the other cases.

Consider the following auxiliary problem

$$
\min _{G_{-} \in \mathbb{G}} \int_{0}^{z_{1}}\left[G_{-}(z)-\left(\lambda_{-}-\mu_{-} M_{-}(z)\right)\right]^{2} w_{-}^{\prime}(z) d z,
$$

which is introduced in Subsection 3.2. The solution to problem (3.8) is summarized in the following lemma. 
Lemma 3.3 i) If $M_{-}(z)$ is from Case 1 , or from Case 2 with $\mathbb{E}_{-}^{z_{1}}(\rho)>M_{-}(0)$, then the optimal solution for problem (3.8) is

$$
G_{-}^{*}(z)=\left[\lambda_{-}-\mu_{-} M_{-}(z)\right] \mathbf{1}_{0<z \leq z_{-}^{*}}+\left[\lambda_{-}-\mu_{-} M_{-}\left(z_{-}^{*}\right)\right] \mathbf{1}_{z_{-}^{*}<z \leq z_{1}},
$$

where $z_{-}^{*}$ is determined by

$$
\int_{z_{-}^{*}}^{z_{1}}\left[M_{-}\left(z_{-}^{*}\right)-M_{-}(z)\right] w_{-}^{\prime}(z) d z=0
$$

In Case 1 , we have $z_{-}^{*} \in\left(\bar{z}_{0_{-}}, z_{0-}\right)$, where $\bar{z}_{0_{-}} \in\left(0, z_{0_{-}}\right)$is the value at which $M_{-}\left(\bar{z}_{0_{-}}\right)=$ $M_{-}\left(z_{1}\right)$. In Case 2 with $\mathbb{E}_{-}^{z_{1}}(\rho)>M_{-}(0)$, we have $z_{-}^{*} \in\left(0, z_{0-}\right)$.

ii) If $M_{-}(z)$ is from Case 3 with $\mathbb{E}_{-}^{z_{1}}(\rho)<M_{-}\left(z_{1}\right)$, or from Case 4, then the optimal solution for problem (3.8) is

$$
G_{-}^{*}(z)=\left[\lambda_{-}-\mu_{-} M_{-}\left(z_{-}^{*}\right)\right] 1_{0<z \leq z_{-}^{*}}+\left[\lambda_{-}-\mu_{-} M_{-}(z)\right] 1_{z_{-}^{*}<z \leq z_{1}},
$$

where $z_{-}^{*}$ is determined by

$$
\int_{0}^{z_{-}^{*}}\left[M_{-}\left(z_{-}^{*}\right)-M_{-}(z)\right] w_{-}^{\prime}(z) d z=0
$$

In Case 3 with $\mathbb{E}_{-}^{z_{1}}(\rho)<M_{-}\left(z_{1}\right)$, we have $z_{-}^{*} \in\left(z_{0-}, z_{1}\right)$. In Case 4, we have $z_{-}^{*} \in$ $\left(z_{0_{-}}, \bar{z}_{0_{-}}\right)$, where $\bar{z}_{0_{-}} \in\left(z_{0_{-}}, z_{1}\right)$ is the value at which $M_{-}\left(\bar{z}_{0-}\right)=M_{-}(0)$.

Proof. The proof of this lemma is similar to that of Lemma 3.2, so we omit the proof here.

If we add the constraint that $G_{-}(z) \geq 0$, and consider problem (3.2), the optimal solution is summarized in the following theorem.

Theorem $3.3 \quad$ i) If $M_{-}(z)$ is from Case 1 , or from Case 2 with $\mathbb{E}_{-}^{z_{1}}(\rho)>M_{-}(0)$, then the optimal solution for problem (3.2) is

$$
G_{-}^{*}(z)=\left[\lambda_{-}-\mu_{-} M_{-}(z)\right]^{+} \mathbf{1}_{0<z \leq z_{-}^{*}}+\left[\lambda_{-}-\mu_{-} M_{-}\left(z_{-}^{*}\right)\right]^{+} \boldsymbol{1}_{z_{-}^{*}<z \leq z_{1}},
$$

where $z_{-}^{*}$ is determined by (3.9).

ii) If $M_{-}(z)$ is from Case 3 with $\mathbb{E}_{-}^{z_{1}}(\rho)<M_{-}\left(z_{1}\right)$, or from Case 4, then the optimal solution for problem (3.2) is

$$
G_{-}^{*}(z)=\left[\lambda_{-}-\mu_{-} M_{-}\left(z_{-}^{*}\right)\right]^{+} \boldsymbol{1}_{0<z \leq z_{-}^{*}}+\left[\lambda_{-}-\mu_{-} M_{-}(z)\right]^{+} \boldsymbol{1}_{z_{-}^{*}<z \leq z_{1}},
$$

where $z_{-}^{*}$ is determined by (3.10).

Proof. The proof of this theorem is similar to that in Theorem 3.2, so we omit the details.

We have given the optimal solution for (3.2) in Theorem 3.3. In order to get the optimal solution for problem (2.12), we need to determine the Lagrange multipliers, $\left(\lambda_{-}, \mu_{-}\right)$. Fortunately, we have solved for the existence and uniqueness of $\left(\lambda_{-}, \mu_{-}\right)$from Proposition 3.3.

Thus we are able to solve for the optimal solution for problem (2.12). We use $M_{-}(z)$ from Case 3 with $\mathbb{E}_{-}^{z_{1}}(\rho)<M_{-}\left(z_{1}\right)$, or from Case 4 , as the main examples in our solution. The conclusions under other cases can be derived using the same process we show in our solution. If 
we assume $M_{-}(z)$ is from Case 3 with $\mathbb{E}_{-}^{z_{1}}(\rho)<M_{-}\left(z_{1}\right)$, or from Case 4 , and insert the optimal solution from Theorem 3.3 into the two constraints from (2.12), we get

$$
\left\{\begin{array}{l}
\int_{0}^{z_{-}^{*}}\left[\lambda_{-}-\mu_{-} M_{-}\left(z_{-}^{*}\right)\right]^{+} w_{-}^{\prime}(z) d z+\int_{z_{-}^{*}}^{z_{1}}\left[\lambda_{-}-\mu_{-} M_{-}(z)\right]^{+} w_{-}^{\prime}(z) d z=a_{+} \\
\int_{0}^{z_{-}^{*}}\left[\lambda_{-}-\mu_{-} M_{-}\left(z_{-}^{*}\right)\right]^{+} F_{\rho}^{-1}(1-z) d z+\int_{z_{-}^{*}}^{z_{1}}\left[\lambda_{-}-\mu_{-} M_{-}(z)\right]^{+} F_{\rho}^{-1}(1-z) d z=y_{+}-y_{0} .
\end{array}\right.
$$

Using Proposition 3.3 and (3.10), we know that (3.11) has the unique solution $\mu_{-}\left(z_{1}, a_{+}, y_{+}\right)>0$. Thus, we are able to get the optimal solution for problem (2.12) which is shown in Theorem 3.3.

\subsection{The optimal solution for problems (2.11) and (2.12) if $y_{0}+a_{+} \mathbb{E}_{-}^{z_{1}}(\rho)<$ $a_{+} \mathbb{E}_{+}^{z_{1}}(\rho)$}

In this subsection, we solve the positive part, problem (2.11), and the negative part, problem (2.12), when $y_{0}+a_{+} \mathbb{E}_{-}^{z_{1}}(\rho)<a_{+} \mathbb{E}_{+}^{z_{1}}(\rho)$. Under this situation, we know from Theorem 3.1 that we only need to consider the parameter interval $y_{+} \in\left(y_{0}+a_{+} \mathbb{E}_{-}^{z_{1}}(\rho), a_{+} \mathbb{E}_{+}^{z_{1}}(\rho)\right)$. Furthermore, we know from Theorem 3.1 that $\mu_{+}>0$ and $\mu_{-}<0$ if $y_{+} \in\left(y_{0}+a_{+} \mathbb{E}_{-}^{z_{1}}(\rho), a_{+} \mathbb{E}_{+}^{z_{1}}(\rho)\right)$. We solve the positive and the negative part problems in Subsection 3.4.1 and Subsection 3.4.2, respectively. The conclusion in this subsection is parallel to the one found in Subsection 3.3. So we omit the proofs and only show the results in the following subsections.

\subsubsection{The optimal solution for the positive part problem (2.11)}

From Proposition 3.1, we know that if $M_{+}(z)$ is from Case 1 with $\mathbb{E}_{+}^{z_{1}}(\rho) \in\left(M_{+}\left(z_{1}\right), M_{+}(1)\right]$, or from Case 4 with $\mathbb{E}_{+}^{z_{1}}(\rho) \in\left[M_{+}\left(z_{1}\right), M_{+}(1)\right)$, then there exists no feasible solution for problem (2.11), so next we consider the optimal solution for problem (3.1) in relation to the other cases.

Lemma 3.4 i) Assume $M_{+}(z)$ is from Case 1 with $\mathbb{E}_{+}^{z_{1}}(\rho)>M_{+}(1)$, or from Case 2, then the optimal solution for (3.4) is

$$
G_{+}^{*}(z)=\left[\lambda_{+}-\mu_{+} M_{+}\left(z_{+}^{*}\right)\right] \mathbf{1}_{z_{1}<z \leq z_{+}^{*}}+\left[\lambda_{+}-\mu_{+} M_{+}(z)\right] \mathbf{1}_{z_{+}^{*}<z \leq 1},
$$

where $z_{+}^{*}$ is determined by

$$
\int_{z_{1}}^{z_{+}^{*}}\left[M_{+}\left(z_{+}^{*}\right)-M_{+}(z)\right] w_{+}^{\prime}(1-z) d z=0 .
$$

In Case 1 with $\mathbb{E}_{+}^{z_{1}}(\rho)>M_{+}(1)$, we have $z_{+}^{*} \in\left[z_{0+}, 1\right)$. In Case 2, we have $z_{+}^{*} \in\left[z_{0+}, \bar{z}_{0+}\right)$, where $\bar{z}_{0+} \in\left[z_{0+}, 1\right)$ is the value at which $M_{+}\left(\bar{z}_{0+}\right)=M_{+}\left(z_{1}\right)$.

ii) Assume $M_{+}(z)$ is from Case 3, or from Case 4 with $\mathbb{E}_{+}^{z_{1}}(\rho)<M_{+}\left(z_{1}\right)$, then the optimal solution for problem (3.4) is

$$
G_{+}^{*}(z)=\left[\lambda_{+}-\mu_{+} M_{+}(z)\right] \mathbf{1}_{z_{1}<z \leq z_{+}^{*}}+\left[\lambda_{+}-\mu_{+} M_{+}\left(z_{+}^{*}\right)\right] \mathbf{1}_{z_{+}^{*}<z \leq 1},
$$

where $z_{+}^{*}$ is determined by

$$
\int_{z_{+}^{*}}^{1}\left[M_{+}\left(z_{+}^{*}\right)-M_{+}(z)\right] w_{+}^{\prime}(1-z) d z=0
$$


In Case 3, we have $z_{+}^{*} \in\left[\bar{z}_{0+}, z_{0+}\right]$, where $\bar{z}_{0+} \in\left(z_{1}, z_{0+}\right)$ is the value at which $M_{+}\left(\bar{z}_{0+}\right)=$ $M_{+}(1)$. In Case 4 with $\mathbb{E}_{+}^{z_{1}}(\rho)<M_{+}\left(z_{1}\right)$, we have $z_{+}^{*} \in\left[z_{1}, z_{0+}\right]$.

Theorem $3.4 \quad$ i) Assume $M_{+}(z)$ is from Case 1 with $\mathbb{E}_{+}^{z_{1}}(\rho)>M_{+}(1)$, or from Case 2, then the optimal solution for (3.1) is

$$
G_{+}^{*}(z)=\left[\lambda_{+}-\mu_{+} M_{+}\left(z_{+}^{*}\right)\right]^{+} \mathbf{1}_{z_{1}<z \leq z_{+}^{*}}+\left[\lambda_{+}-\mu_{+} M_{+}(z)\right]^{+} \mathbf{1}_{z_{+}^{*}<z \leq 1},
$$

where $z_{+}^{*}$ is determined by (3.12).

ii) Assume $M_{+}(z)$ is from Case 3, or from Case 4 with $\mathbb{E}_{+}^{z_{1}}(\rho)<M_{+}\left(z_{1}\right)$, then the optimal solution for problem (3.1) is

$$
G_{+}^{*}(z)=\left[\lambda_{+}-\mu_{+} M_{+}(z)\right]^{+} \mathbf{1}_{z_{1}<z \leq z_{+}^{*}}+\left[\lambda_{+}-\mu_{+} M_{+}\left(z_{+}^{*}\right)\right]^{+} \mathbf{1}_{z_{+}^{*}<z \leq 1},
$$

where $z_{+}^{*} \in\left[\bar{z}_{0+}, z_{0+}\right]$ is determined by (3.13).

Next we solve problem (2.11). We take $M_{+}(z)$ from Case 3 , or from Case 4 with $\mathbb{E}_{+}^{z_{1}}(\rho)<$ $M_{+}\left(z_{1}\right)$, as the main examples in our solution. The conclusions under other cases can be derived using the same process we show in our solution. If we assume $M_{+}(z)$ is from Case 3 , or from Case 4 with $\mathbb{E}_{+}^{z_{1}}(\rho)<M_{+}\left(z_{1}\right)$, and insert the optimal solution from Theorem 3.4 into the two constraints from (2.11), we get

$$
\left\{\begin{array}{l}
\int_{z_{1}}^{z_{+}^{*}}\left[\lambda_{+}-\mu_{+} M_{+}(z)\right]^{+} w_{+}^{\prime}(1-z) d z+\int_{z_{+}^{*}}^{1}\left[\lambda_{+}-\mu_{+} M_{+}\left(z_{+}^{*}\right)\right]^{+} w_{+}^{\prime}(1-z) d z=a_{+} \\
\int_{z_{1}}^{z_{+}^{*}}\left[\lambda_{+}-\mu_{+} M_{+}(z)\right]^{+} F_{\rho}^{-1}(1-z) d z+\int_{z_{+}^{*}}^{1}\left[\lambda_{+}-\mu_{+} M_{+}\left(z_{+}^{*}\right)\right]^{+} F_{\rho}^{-1}(1-z) d z=y_{+} .
\end{array}\right.
$$

Using Proposition 3.3 and (3.13), we know that (3.14) has the unique solution $\mu_{+}\left(z_{1}, a_{+}, y_{+}\right)>0$ and $\lambda_{+}\left(z_{1}, a_{+}, y_{+}\right)$. Thus, we are able to get the optimal solution for problem (2.11) which is shown in Theorem 3.4 .

So far, we have gotten the optimal solution for the positive part problem (2.11) when $y_{0}+a_{+} \mathbb{E}_{-}^{z_{1}}(\rho)<a_{+} \mathbb{E}_{+}^{z_{1}}(\rho)$. In Subsection 3.4.2, we solve for the negative part problem $(2.12)$ under the same situation.

\subsubsection{The optimal solution for the negative part problem (2.12)}

From Proposition 3.2, we know that if $M_{-}(z)$ is from Case 1 with $\mathbb{E}_{-}^{z_{1}}(\rho) \in\left(M_{-}(0), M_{-}\left(z_{1}\right)\right]$, or from Case 4 with $\mathbb{E}_{-}^{z_{1}}(\rho) \in\left[M_{-}(0), M_{-}\left(z_{1}\right)\right)$, then there exists no feasible solution for problem (2.12), so next we consider the optimal solution for problem (3.2) in relation to the other cases.

Lemma 3.5 i) If $M_{-}(z)$ is from Case 1 with $\mathbb{E}_{-}^{z_{1}}(\rho)>M_{-}\left(z_{1}\right)$, or from Case 2, then the optimal solution for problem (3.8) is

$$
G_{-}^{*}(z)=\left[\lambda_{-}-\mu_{-} M_{-}\left(z_{-}^{*}\right)\right] 1_{0<z \leq z_{-}^{*}}+\left[\lambda_{-}-\mu_{-} M_{-}(z)\right] \boldsymbol{1}_{z_{-}^{*}<z \leq z_{1}},
$$

where $z_{-}^{*}$ is determined by

$$
\int_{0}^{z_{-}^{*}}\left[M_{-}\left(z_{-}^{*}\right)-M_{-}(z)\right] w_{-}^{\prime}(z) d z=0
$$

In Case 1 with $\mathbb{E}_{-}^{z_{1}}(\rho)>M_{-}\left(z_{1}\right)$, we have $z_{-}^{*} \in\left(z_{0-}, z_{1}\right)$. In Case 2, we have $z_{-}^{*} \in$ $\left(z_{0-}, \bar{z}_{0-}\right)$, where $\bar{z}_{0-} \in\left(z_{0-}, z_{1}\right)$ is the value at which $M_{-}\left(\bar{z}_{0-}\right)=M_{-}(0)$. 
ii) If $M_{-}(z)$ is from Case 3, or from Case 4 with $\mathbb{E}_{-}^{z_{1}}(\rho)<M_{-}(0)$, then the optimal solution for problem (3.8) is

$$
G_{-}^{*}(z)=\left[\lambda_{-}-\mu_{-} M_{-}(z)\right] 1_{0<z \leq z_{-}^{*}}+\left[\lambda_{-}-\mu_{-} M_{-}\left(z_{-}^{*}\right)\right] 1_{z_{-}^{*}<z \leq z_{1}},
$$

where $z_{-}^{*}$ is determined by

$$
\int_{z_{-}^{*}}^{z_{1}}\left[M_{-}\left(z_{-}^{*}\right)-M_{-}(z)\right] w_{-}^{\prime}(z) d z=0
$$

In Case 3 , we have $z_{-}^{*} \in\left(\bar{z}_{0_{-}}, z_{0_{-}}\right)$, where $\bar{z}_{0_{-}} \in\left(0, z_{0_{-}}\right)$is the value at which $M_{-}\left(\bar{z}_{0_{-}}\right)=$ $M_{-}\left(z_{1}\right)$. In Case 4 with $\mathbb{E}_{-}^{z_{1}}(\rho)<M_{-}\left(z_{1}\right)$, we have $z_{-}^{*} \in\left(0, z_{0_{-}}\right)$.

Theorem 3.5 i) If $M_{-}(z)$ is from Case 1 with $\mathbb{E}_{-}^{z_{1}}(\rho)>M_{-}\left(z_{1}\right)$, or from Case 2, then the optimal solution for problem (3.2) is

$$
G_{-}^{*}(z)=\left[\lambda_{-}-\mu_{-} M_{-}\left(z_{-}^{*}\right)\right]^{+} \boldsymbol{1}_{0<z \leq z_{-}^{*}}+\left[\lambda_{-}-\mu_{-} M_{-}(z)\right]^{+} \boldsymbol{1}_{z_{-}^{*}<z \leq z_{1}},
$$

where $z_{-}^{*}$ is determined by (3.15).

ii) If $M_{-}(z)$ is from Case 3 , or from Case 4 with $\mathbb{E}_{-}^{z_{1}}(\rho)<M_{-}(0)$, then the optimal solution for problem (3.2) is

$$
G_{-}^{*}(z)=\left[\lambda_{-}-\mu_{-} M_{-}(z)\right]^{+} \mathbf{1}_{0<z \leq z_{-}^{*}}+\left[\lambda_{-}-\mu_{-} M_{-}\left(z_{-}^{*}\right)\right]^{+} \boldsymbol{1}_{z_{-}^{*}<z \leq z_{1}},
$$

where $z_{-}^{*}$ is determined by (3.16).

Next we solve problem (2.12). We take $M_{-}(z)$ from Case 3 , or from Case 4 with $\mathbb{E}_{-}^{z_{1}}(\rho)<$ $M_{-}(0)$, as the main examples in our solution. The conclusions under other cases can be derived using the same methods shown in our solution. Assuming $M_{-}(z)$ is from Case 3, or from Case 4 with $\mathbb{E}_{-}^{z_{1}}(\rho)<M_{-}(0)$, we insert the optimal solution in Theorem 3.5 into the two constraints in $(2.12)$, and get

$$
\left\{\begin{array}{l}
\int_{0}^{z_{-}^{*}}\left[\lambda_{-}-\mu_{-} M_{-}(z)\right]^{+} w_{-}^{\prime}(z) d z+\int_{z_{-}^{*}}^{z_{1}}\left[\lambda_{-}-\mu_{-} M_{-}\left(z_{-}^{*}\right)\right]^{+} w_{-}^{\prime}(z) d z=a_{+} \\
\int_{0}^{z_{-}^{*}}\left[\lambda_{-}-\mu_{-} M_{-}(z)\right]^{+} F_{\rho}^{-1}(1-z) d z+\int_{z_{-}^{*}}^{z_{1}}\left[\lambda_{-}-\mu_{-} M_{-}\left(z_{-}^{*}\right)\right]^{+} F_{\rho}^{-1}(1-z) d z=y_{+}-y_{0} .
\end{array}\right.
$$

Using Proposition 3.3 and (3.16), we know that (3.17) has the unique solution $\mu_{-}\left(z_{1}, a_{+}, y_{+}\right)<0$. Thus we are able to get the optimal solution for problem (2.12) which is shown in Theorem 3.5.

\section{The optimal attainable wealth}

In the above section, we solved the positive and negative part problems in Step 1. In this section, we talk about problem (2.13) from Step 2. We give the optimal values $v_{+}\left(z_{1}, a_{+}, y_{+}\right)$and $v_{-}\left(z_{1}, a_{+}, y_{+}\right)$of the positive and negative part problems when $a_{+} \mathbb{E}_{+}^{z_{1}}(\rho) \leq y_{+} \leq y_{0}+a_{+} \mathbb{E}_{-}^{z_{1}}(\rho)$ (see Subsection 3.3), then $\mu_{+}\left(z_{1}, a_{+}, y_{+}\right) \leq 0, \mu_{-}\left(z_{1}, a_{+}, y_{+}\right) \geq 0$. The result for other cases can be derived using the same method we show in this section.

We only consider $M_{+}(z)$ from Case 3 with $\mathbb{E}_{+}^{z_{1}}(\rho)<M_{+}(1)$, or from Case 4 (see Theorem 3.2 ii)). The results for other cases can be derived similarly, so we omit them here. If $M_{+}(z)$ is 
from Case 3 with $\mathbb{E}_{+}^{z_{1}}(\rho)<M_{+}(1)$, or from Case 4, then the optimal value of the positive part, problem (2.11), is

$$
\begin{aligned}
v_{+}\left(z_{1}, a_{+}, y_{+}\right)= & \int_{z_{1}}^{z_{+}^{*}}\left[\lambda_{+}-\mu_{+} M_{+}\left(z_{+}^{*}\right)\right]\left[\lambda_{+}-\mu_{+} M_{+}\left(z_{+}^{*}\right)\right]^{+} w_{+}^{\prime}(1-z) d z \\
& +\int_{z_{+}^{*}}^{1}\left[\lambda_{+}-\mu_{+} M_{+}(z)\right]\left[\lambda_{+}-\mu_{+} M_{+}(z)\right]^{+} w_{+}^{\prime}(1-z) d z \\
= & \lambda_{+} a_{+}-\mu_{+} y_{+},
\end{aligned}
$$

in which we use $\left(c^{+}\right)^{2}=c c^{+}$for any c, and equations (3.6)-(3.7).

Moreover, if $M_{-}(z)$ is from Case 3 with $\mathbb{E}_{-}^{z_{1}}(\rho)<M_{-}\left(z_{1}\right)$, then the optimal value of the negative part problem $(2.12)$ is

$$
v_{-}\left(z_{1}, a_{+}, y_{+}\right)=\lambda_{-} a_{+}-\mu_{-}\left(y_{+}-y_{0}\right) .
$$

It is not difficult to verify that $v_{+}\left(z_{1}, a_{+}, y_{+}\right)$and $v_{-}\left(z_{1}, a_{+}, y_{+}\right)$have the same form as above when other cases are considered. At this point, problem (2.13) becomes

$$
\begin{aligned}
& \min \lambda_{+}\left(z_{1}, a_{+}, y_{+}\right) a_{+}-\mu_{+}\left(z_{1}, a_{+}, y_{+}\right) y_{+}+\lambda_{-}\left(z_{1}, a_{+}, y_{+}\right) a_{+}-\mu_{-}\left(z_{1}, a_{+}, y_{+}\right)\left(y_{+}-y_{0}\right) \\
& \text { subject to }\left\{\begin{array}{l}
z_{1} \in(0,1) \\
a_{+}>0 \\
y_{+}>0
\end{array}\right.
\end{aligned}
$$

Notice that it is not easy to give the explicit solutions for $\left(\lambda_{+}, \mu_{+}\right)$and $\left(\lambda_{-}, \mu_{-}\right)$. Due to this, we cannot derive the explicit forms of $v_{+}\left(z_{1}, a_{+}, y_{+}\right)$and $v_{-}\left(z_{1}, a_{+}, y_{+}\right)$. Instead, we derive certain properties about the optimal value.

\subsection{The optimal quantile when $a_{+} \mathbb{E}_{+}^{z_{1}}(\rho) \leq y_{0}+a_{+} \mathbb{E}_{-}^{z_{1}}(\rho)$}

Under the situation $a_{+} \mathbb{E}_{+}^{z_{1}}(\rho) \leq y_{0}+a_{+} \mathbb{E}_{-}^{z_{1}}(\rho)$, we only need to consider $y_{+} \in\left[a_{+} \mathbb{E}_{+}^{z_{1}}(\rho), y_{0}+\right.$ $\left.a_{+} \mathbb{E}_{-}^{z_{1}}(\rho)\right]$. From Theorem 3.1, we know that if the optimal values of parameters $\left(z_{1}, a_{+}, y_{+}\right)$, for problem (4.1) satisfy $a_{+} \mathbb{E}_{+}^{z_{1}}(\rho) \leq y_{+} \leq y_{0}+a_{+} \mathbb{E}_{-}^{z_{1}}(\rho)$, then $\mu_{+} \leq 0, \mu_{-} \geq 0$. This produces the following theorems.

Theorem $4.1 \quad$ i) Assume $M_{+}(z)$ is from Case 3 with $\mathbb{E}_{+}^{z_{1}}(\rho)<M_{+}(1)$ or from Case 4. If $\left(z_{1}, a_{+}, y_{+}\right)$from problem (4.1) satisfy

$$
\mathbb{E}_{+}^{z_{1}}(\rho)<\frac{y_{+}}{a_{+}}<\frac{\int_{z_{+}^{*}}^{1} M_{+}(z)\left[M_{+}(z)-M_{+}\left(z_{+}^{*}\right)\right] \frac{w_{+}^{\prime}(1-z)}{\int_{z_{1}}^{1} w_{+}^{\prime}(1-x) d x} d z}{\int_{z_{+}^{*}}^{1}\left[M_{+}(z)-M_{+}\left(z_{+}^{*}\right)\right] \frac{w_{+}^{\prime}(1-z)}{\int_{z_{1}}^{1} w_{+}^{\prime}(1-x) d x} d z},
$$

we see that $\lambda_{+}-\mu_{+} M_{+}\left(z_{+}^{*}\right)>0$, and that the optimal solution to (2.11) is

$$
G_{+}^{*}(z)=\left[\lambda_{+}-\mu_{+} M_{+}\left(z_{+}^{*}\right)\right] \mathbf{1}_{z_{1}<z \leq z_{+}^{*}}+\left[\lambda_{+}-\mu_{+} M_{+}(z)\right] \mathbf{1}_{z_{+}^{*}<z \leq 1},
$$

where $z_{+}^{*}$ is determined by (3.6) and $\left(\lambda_{+}, \mu_{+}\right)$is given by 


$$
\left\{\begin{array}{l}
\mu_{+}=\frac{\frac{y_{+}-a_{+} \mathbb{E}_{+}^{z_{1}}(\rho)}{\int_{z_{1}}^{1} w_{+}^{\prime}(1-z) d z}}{\left\{\int_{z_{+}^{*}}^{1}\left[M_{+}\left(z_{+}^{*}\right)-M_{+}(z)\right] \frac{w_{+}^{\prime}(1-z)}{\int_{z_{1}}^{1} w_{+}^{\prime}(1-x) d x} d z\right\}^{2}-\int_{z_{+}^{*}}^{1}\left[M_{+}\left(z_{+}^{*}\right)-M_{+}(z)\right]^{2} \frac{w_{+}^{\prime}(1-z)}{\int_{z_{1}}^{1} w_{+}^{\prime}(1-x) d x} d z}<0 \\
\lambda_{+}=\frac{a_{+}}{\int_{z_{1}}^{1} w_{+}^{\prime}(1-z) d z}+\mu_{+} \mathbb{E}_{+}^{z_{+}}(\rho) .
\end{array}\right.
$$

Moreover, the optimal value of problem (2.11) is

$$
\begin{aligned}
= & \frac{v_{+}\left(z_{1}, a_{+}, y_{+}\right)}{\left.\int_{z_{+}^{*}}^{1}\left[M_{+}\left(z_{+}^{*}\right)-M_{+}(z)\right]^{2} \frac{w_{+}^{\prime}(1-z)}{\int_{z_{1}}^{1} w_{+}^{\prime}(1-x) d x} d z-\left\{\int_{z_{+}^{*}}^{1}\left[M_{+}\left(z_{+}^{*}\right)-M_{+}(z)\right] \frac{w_{+}^{\prime}(1-z)}{\int_{z_{1}}^{1} w_{+}^{\prime} w_{+}^{\prime}(1-x) d x} d z\right\}^{z_{1}}(\rho)\right]^{2}} \\
& +\frac{a_{+}^{2}}{\int_{z_{1}}^{1} w_{+}^{\prime}(1-z) d z} .
\end{aligned}
$$

ii) Assume $M_{-}(z)$ is from Case 3 with $\mathbb{E}_{-}^{z_{1}}(\rho)<M_{-}\left(z_{1}\right)$, or from Case 4. If $\left(z_{1}, a_{+}, y_{+}\right)$from problem (4.1) satisfy

$\mathbb{E}_{-}^{z_{1}}(\rho)>\frac{y_{+}-y_{0}}{a_{+}}>\frac{\mathbb{E}_{-}^{z_{1}}(\rho)\left[M_{-}\left(z_{-}^{*}\right)-M_{-}\left(z_{1}\right)\right]+\int_{z_{-}^{*}}^{z_{1}}\left[M_{-}(z)-M_{-}\left(z_{-}^{*}\right)\right] M_{-}(z) \frac{w_{-}^{\prime}(z)}{\int_{0}^{z_{1}} w_{-}^{\prime}(x) d x} d z}{\mathbb{E}_{-}^{z_{1}}(\rho)-M_{-}\left(z_{1}\right)}$,

we see that $\lambda_{-}-\mu_{-} M_{-}\left(z_{1}\right)>0$, and that the optimal solution for (2.12) is

$$
G_{-}^{*}(z)=\left[\lambda_{-}-\mu_{-} M_{-}\left(z_{-}^{*}\right)\right] \mathbf{1}_{0<z \leq z_{-}^{*}}+\left[\lambda_{-}-\mu_{-} M_{-}(z)\right] \mathbf{1}_{z_{-}^{*}<z \leq z_{1}},
$$

where $z_{-}^{*}$ is determined by (3.10) and $\left(\lambda_{-}, \mu_{-}\right)$is given by

$$
\left\{\begin{array}{l}
\mu_{-}=\frac{\frac{y_{+}-y_{0}-a_{+} \mathbb{E}_{-}^{z_{1}}(\rho)}{\int_{0}^{z_{1}} w_{-}^{\prime}(z) d z}}{\left\{\int_{z_{-}^{*}}^{z_{1}}\left[M_{-}\left(z_{-}^{*}\right)-M_{-}(z)\right] \frac{w_{-}^{\prime}(z)}{\int_{0}^{z_{1}} w_{-}^{\prime}(x) d x} d z\right\}^{2}-\int_{z_{-}^{*}}^{z_{1}}\left[M_{-}\left(z_{-}^{*}\right)-M_{-}(z)\right]^{2} \frac{w_{-}^{\prime}(z)}{\int_{0}^{z_{1}} w_{-}^{\prime}(x) d x} d z}>0 \\
\lambda_{-}=\frac{a_{+}}{\int_{0}^{z_{1}} w_{-}^{\prime}(z) d z}+\mu_{-} \mathbb{E}_{-}^{z_{1}}(\rho) .
\end{array}\right.
$$

Moreover, the optimal value of problem (2.12) is

$$
\begin{aligned}
& v_{-}\left(z_{1}, a_{+}, y_{+}\right) \\
= & \frac{\frac{\left[y_{+}-y_{0}-a_{+} \mathbb{E}_{-}^{z_{1}}(\rho)\right]^{2}}{\int_{0}^{z_{1}} w_{-}^{\prime}(z) d z}}{\int_{z_{-}^{*}}^{z_{1}}\left[M_{-}\left(z_{-}^{*}\right)-M_{-}(z)\right]^{2} \frac{w_{-}^{\prime}(z)}{\int_{0}^{z_{1}} w_{-}^{\prime}(x) d x} d z-\left\{\int_{z_{-}^{*}}^{z_{1}}\left[M_{-}\left(z_{-}^{*}\right)-M_{-}(z)\right] \frac{w_{-}^{\prime}(z)}{\int_{0}^{z_{1}} w_{-}^{\prime}(x) d x} d z\right\}^{2}} \\
& +\frac{a_{+}^{2}}{\int_{0}^{z_{1}} w_{-}^{\prime}(z) d z} .
\end{aligned}
$$




\section{Proof.}

i) Set

$$
\left\{\begin{array}{l}
\mu_{+}=\frac{\frac{y_{+}-a_{+} \mathbb{E}_{+}^{z_{1}}(\rho)}{\int_{z_{1}}^{1} w_{+}^{\prime}(1-z) d z}}{\left\{\int_{z_{+}^{*}}^{1}\left[M_{+}\left(z_{+}^{*}\right)-M_{+}(z)\right] \frac{w_{+}^{\prime}(1-z)}{\int_{z_{1}}^{1} w_{+}^{\prime}(1-x) d x} d z\right\}^{2}-\int_{z_{+}^{*}}^{1}\left[M_{+}\left(z_{+}^{*}\right)-M_{+}(z)\right]^{2} \frac{w_{+}^{\prime}(1-z)}{\int_{z_{1}}^{1} w_{+}^{\prime}(1-x) d x} d z} \\
\lambda_{+}=\frac{a_{+}}{\int_{z_{1}}^{1} w_{+}^{\prime}(1-z) d z}+\mu_{+} \mathbb{E}_{+}^{z_{1}}(\rho),
\end{array}\right.
$$

which makes it easy to verify that $\left(\lambda_{+}, \mu_{+}\right)$satisfy

$$
\left\{\begin{array}{l}
\int_{z_{1}}^{z_{+}^{*}}\left[\lambda_{+}-\mu_{+} M_{+}\left(z_{+}^{*}\right)\right] w_{+}^{\prime}(1-z) d z+\int_{z_{+}^{*}}^{1}\left[\lambda_{+}-\mu_{+} M_{+}(z)\right] w_{+}^{\prime}(1-z) d z=a_{+} \\
\int_{z_{1}}^{z_{+}^{*}}\left[\lambda_{+}-\mu_{+} M_{+}\left(z_{+}^{*}\right)\right] F_{\rho}^{-1}(1-z) d z+\int_{z_{+}^{*}}^{1}\left[\lambda_{+}-\mu_{+} M_{+}(z)\right] F_{\rho}^{-1}(1-z) d z=y_{+} .
\end{array}\right.
$$

Because of equation (4.2) and the fact that

$$
\left\{\int_{z_{+}^{*}}^{1}\left[M_{+}\left(z_{+}^{*}\right)-M_{+}(z)\right] \frac{w_{+}^{\prime}(1-z)}{\int_{z_{1}}^{1} w_{+}^{\prime}(1-x) d x} d z\right\}^{2}-\int_{z_{+}^{*}}^{1}\left[M_{+}\left(z_{+}^{*}\right)-M_{+}(z)\right]^{2} \frac{w_{+}^{\prime}(1-z)}{\int_{z_{1}}^{1} w_{+}^{\prime}(1-x) d x} d z<0,
$$

we see that $\mu_{+}<0$, and

$$
\begin{aligned}
& \lambda_{+}-\mu_{+} M_{+}\left(z_{+}^{*}\right)=\frac{1}{\int_{z_{1}}^{1} w_{+}^{\prime}(1-z) d z} \times \\
& \frac{y_{+} \int_{z_{+}^{*}}^{1}\left[M_{+}(z)-M_{+}\left(z_{+}^{*}\right)\right] \frac{w_{+}^{\prime}(1-z)}{\int_{z_{1}}^{1} w_{+}^{\prime}(1-x) d x} d z-a_{+} \int_{z_{+}^{*}}^{1} M_{+}(z)\left[M_{+}(z)-M_{+}\left(z_{+}^{*}\right)\right] \frac{w_{+}^{\prime}(1-z)}{\int_{z_{1}}^{1} w_{+}^{\prime}(1-x) d x} d z}{\left\{\int_{z_{+}^{*}}^{1}\left[M_{+}\left(z_{+}^{*}\right)-M_{+}(z)\right] \frac{w_{+}^{\prime}(1-z)}{\int_{z_{1}}^{1} w_{+}^{\prime}(1-x) d x} d z\right\}^{2}-\int_{z_{+}^{*}}^{1}\left[M_{+}\left(z_{+}^{*}\right)-M_{+}(z)\right]^{2} \frac{w_{+}^{\prime}(1-z)}{\int_{z_{1}}^{1} w_{+}^{\prime}(1-x) d x} d z}>0 .
\end{aligned}
$$

Set

$$
G_{+}^{*}(z)=\left[\lambda_{+}-\mu_{+} M_{+}\left(z_{+}^{*}\right)\right] \mathbf{1}_{z_{1}<z \leq z_{+}^{*}}+\left[\lambda_{+}-\mu_{+} M_{+}(z)\right] \mathbf{1}_{z_{+}^{*}<z \leq 1},
$$

then $\left(\lambda_{+}, \mu_{+}\right)$satisfy (3.7) and $G_{+}^{*}(z)$ satisfies the constraints in (2.11). According to the result in Subsection 3.3.1, $G_{+}^{*}(z)$ is the optimal value of problem (2.11). After some simple calculation, the optimal value of problem (2.11) can be derived.

ii) The proof of ii) is similar to that of i). So we omit it here.

Remark 4.1 Because the optimal solution for problem (2.8) is $G^{*}(z)=G_{+}^{*}(z)-G_{-}^{*}(z)$, there is a jump between the positive and the negative parts of the optimal solution for problem (2.8). It follows that there is also a jump in the optimal solution for problems (2.7) and (2.5) as well. The jump in the optimal terminal wealth structure is a difference between the behavioral and the classical mean-variance setting. The reason is that we put the probability distortion functions $w_{+}(\cdot)$ and $w_{-}(\cdot)$ on positive wealth $X^{+}$and negative wealth $X^{-}$, respectively. If we set $w_{+}(z)=w_{-}(z)=z$, the behavioral mean and variance of $X$ return to the conventional mean and variance of $X$. Then the result in this paper return to the result in classical mean-variance setting. 


\subsection{The optimal quantile when $y_{+} \in\left(y_{0}+a_{+} \mathbb{E}_{-}^{z_{1}}(\rho), a_{+} \mathbb{E}_{+}^{z_{1}}(\rho)\right)$}

Theorem 4.2 i) Assume $M_{+}(z)$ is from Case 3, or from Case 4 with $\mathbb{E}_{+}^{z_{1}}(\rho)<M_{+}\left(z_{1}\right)$. If the optimal values of $\left(z_{1}, a_{+}, y_{+}\right)$for problem (4.1) satisfy

$$
\mathbb{E}_{+}^{z_{1}}(\rho)>\frac{y_{+}}{a_{+}}>\frac{\mathbb{E}_{+}^{z_{1}}(\rho)\left[M_{+}\left(z_{+}^{*}\right)-M_{+}\left(z_{1}\right)\right]-\int_{z_{1}}^{z_{+}^{*}}\left[M_{+}\left(z_{+}^{*}\right)-M_{+}(z)\right] M_{+}(z) \frac{w_{+}^{\prime}(1-z)}{\int_{z_{1}}^{1} w_{+}^{\prime}(1-x) d x} d z}{\mathbb{E}_{+}^{z_{1}}(\rho)-M_{+}\left(z_{1}\right)}
$$

we have $\lambda_{+}-\mu_{+} M_{+}\left(z_{1}\right)>0$ and the optimal solution for (2.11) is

$$
G_{+}^{*}(z)=\left[\lambda_{+}-\mu_{+} M_{+}(z)\right] \mathbf{1}_{z_{1}<z \leq z_{+}^{*}}+\left[\lambda_{+}-\mu_{+} M_{+}\left(z_{+}^{*}\right)\right] \mathbf{1}_{z_{+}^{*}<z \leq 1} .
$$

This shows that there may exist a jump between the positive and the negative parts of the optimal solution for problem (2.8). It follows that there is also a jump in the optimal solution for problems (2.7) and (2.5) as well.

ii) Assume $M_{-}(z)$ is from Case 3, or from Case 4 with $\mathbb{E}_{-}^{z_{1}}(\rho)<M_{-}(0)$. If the optimal values of parameters $\left(z_{1}, a_{+}, y_{+}\right)$for problem (4.1) satisfy

$$
\mathbb{E}_{-}^{z_{1}}(\rho)<\frac{y_{+}-y_{0}}{a_{+}}<\frac{\int_{z_{-}^{*}}^{z_{1}} M_{-}(z)\left[M_{-}(z)-M_{-}\left(z_{-}^{*}\right)\right] \frac{w_{-}^{\prime}(z)}{\int_{0}^{z_{1}} w_{-}^{\prime}(x) d x} d z}{\int_{z_{-}^{*}}^{z_{1}}\left[M_{-}(z)-M_{-}\left(z_{-}^{*}\right)\right] \frac{w_{-}^{\prime}(z)}{\int_{0}^{z_{1}} w_{-}^{\prime}(x) d x} d z}
$$

we have $\lambda_{-}-\mu_{-} M_{-}\left(z_{-}^{*}\right)>0$ and the optimal solution to (2.12) is

$$
G_{-}^{*}(z)=\left[\lambda_{-}-\mu_{-} M_{-}(z)\right] \mathbf{1}_{0<z \leq z_{-}^{*}}+\left[\lambda_{-}-\mu_{-} M_{-}\left(z_{-}^{*}\right)\right] \mathbf{1}_{z_{-}^{*}<z \leq z_{1}} .
$$

This shows that there may exist a jump between the positive and the negative parts of the optimal solution for problem (2.8). It follows that there is also a jump in the optimal solution for problems (2.7) and (2.5) as well.

Proof. The proof for this theorem is similar to the proof of Theorem 4.1. So we omit it here. $\square$

\subsection{Discussion of an extreme case of the optimal attainable wealth}

In this subsection, we show that under some extreme case, the loss of the investor will go to $\infty$. For example, assume $M_{-}(z)$ is from Case 3 and $M_{-}(0)=+\infty$. If the optimal values of parameters $\left(z_{1}, a_{+}, y_{+}\right)$for problem (4.1) satisfy $\mathbb{E}_{-}^{z_{1}}(\rho)<\frac{y_{+}-y_{0}}{a_{+}}$and then $\mu_{-}<0$, the optimal solution for $(2.12)$ is

$$
G_{-}^{*}(z)=\left[\lambda_{-}-\mu_{-} M_{-}(z)\right]^{+} \mathbf{1}_{0<z \leq z_{-}^{*}}+\left[\lambda_{-}-\mu_{-} M_{-}\left(z_{-}^{*}\right)\right]^{+} \mathbf{1}_{z_{-}^{*}<z \leq z_{1}},
$$

which means that $G_{-}^{*}(0)=+\infty$, then the loss of the investor will go to $\infty$, i.e., the bad state will go to $-\infty$.

From the conclusions we derived in this paper, we know that, if $M_{-}(z)$ is from other cases, i.e., Case 1, 2, 4 or Case 3 with $M_{-}(0)<+\infty$, then the bad state cannot go to $-\infty$. 


\section{$5 \quad$ Examples}

In this section, we give some examples to illustrate our results. We show that some commonly used probability distortions are included in the four cases we considered above.

Example 5.1 We assume $w_{+}(z)=z^{\gamma}$ for some $\gamma \in(0,1]$. Because $F_{\rho}^{-1}(1-z)$ is decreasing for $z \in(0,1)$ for any $\rho, M_{+}(z)=\frac{F_{\rho}^{-1}(1-z)}{w_{+}^{\prime}(1-z)}$ is decreasing for $z \in(0,1)$. Notice that this form of $M_{+}(z)$ is included in Case 3 as $z_{0+}=1$.

Moreover, we assume $w_{-}(z)=z^{\gamma}$ for some $\gamma \in(0,1]$ and $\rho$ follows a log-normal distribution, i.e., $F_{\rho}(x)=\Phi\left(\frac{\ln x-\tilde{\eta}}{\tilde{\sigma}}\right)$, where $\Phi(\cdot)$ is the cumulative distribution function of the standard normal distribution. It is not difficult to verify that $M_{-}(z)$ is increasing across $\left(0, z_{0}\right)$ and decreasing across $\left(z_{0}, 1\right)$ for some $z_{0} \in[0,1]$ and $M_{-}(1)=0$. This $M_{-}(z)$ is included in Case 2 .

A reversed S-shaped probability distortion has been used and studied by many authors, see, e.g., Tversky and Kahneman (1992), Jin and Zhou (2008), Bi et al. (2013), and so on. For a reversed S-shaped distortion $w(x), w^{\prime}(x)>1$ around both $x=0$ and $x=1$. This implies that the reversed S-shaped distortion puts higher weights on both very good and very bad outcomes. In other words, the agent exaggerates the small probabilities of both very good and very bad scenarios.

Example 5.2 Take

$$
w_{+}(z)=\frac{z^{\gamma}}{\left[z^{\gamma}+(1-z)^{\gamma}\right]^{\frac{1}{\gamma}}},
$$

for some $\gamma \in(0,1)$, which is the reversed S-shaped distortion, making $w_{+}(z)$ is concave initially and then convex. $\rho$ also follows the log-normal distribution, i.e., $F_{\rho}(x)=\Phi\left(\frac{\ln x-\tilde{\eta}}{\tilde{\sigma}}\right)$.

If we let $\gamma=0.7, \tilde{\eta}=0.1$ and $\tilde{\sigma}=0.4$, and then use the mathematical software MATLAB, we can draw the picture of $M_{+}(z)$ in Figure 5. We can see from Figure 5 that $M_{+}(z)$ increases first but later starts to decrease. We can also see that $M_{+}(z)$ attains its maximum at some value $z_{0}$. Moreover, $M_{+}(1)=\frac{F_{\rho}^{-1}(0)}{w_{+}^{\prime}(0)}=0 \leq M_{+}(0)$. Thus, this form of $M_{+}(z)$ is included in Case 2. Actually, $M_{+}(z)$ is included in Case 2 for $\forall \gamma \in(0,1)$.

Meanwhile, take

$$
w_{-}(z)=\frac{z^{\gamma}}{\left[z^{\gamma}+(1-z)^{\gamma}\right]^{\frac{1}{\gamma}}},
$$

for some $\gamma \in(0,1)$, and $\rho$ also follows a log-normal distribution, i.e., $F_{\rho}(x)=\Phi\left(\frac{\ln x-\tilde{\eta}}{\tilde{\sigma}}\right)$.

If we let $\gamma=0.7, \tilde{\eta}=0.1$ and $\tilde{\sigma}=0.4$, and then use MATLAB, we can draw the picture of $M_{-}(z)$ in Figure 6. We can see from the picture that $M_{-}(z)$ increases first but later starts to decrease. We can also see that $M_{-}(z)$ attains its maximum at some value $z_{0}$. Moreover, $M_{-}(0)=\frac{F_{\rho}^{-1}(1)}{w_{-}^{\prime}(0)}=0 \leq M_{-}(1)$. Thus, this form of $M_{-}(z)$ is included in Case 1. Actually, $M_{-}(z)$ is included in Case 1 for $\forall \gamma \in(0,1)$.

\section{The efficient frontier and the efficient strategy}

In this section, we show the efficient frontier and the efficient strategy of the initial behavioral mean-variance problem (2.2). 


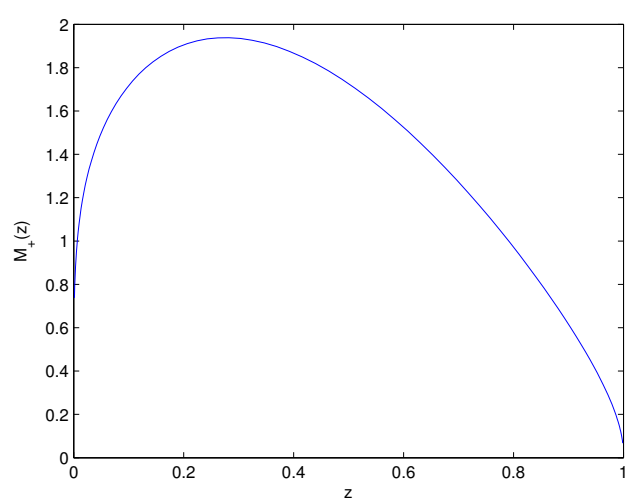

Fig. 5. $M_{+}(z)$ in Example 5.2

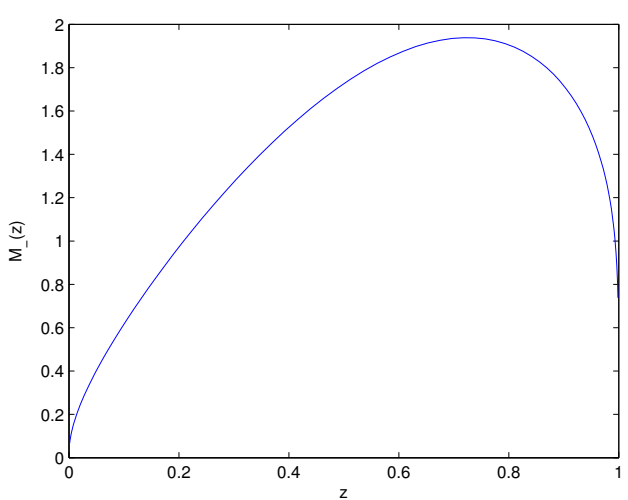

Fig. 6. $M_{-}(z)$ in Example 5.2

From Sections 2.4-2.5, we know that the optimal solution for the quantile problem (2.8) is

$$
G^{*}(z)=G_{+}^{*}(z) \mathbf{1}_{z_{1}^{*}<z \leq 1}-G_{-}^{*}(z) \mathbf{1}_{0<z \leq z_{1}^{*}},
$$

where $G_{+}^{*}(z)$ and $G_{-}^{*}(z)$ are the optimal solutions for the positive part problem (2.9), and the negative part problem (2.10), respectively, and $z_{1}^{*}$ is optimal for problem (4.1). It follows that the optimal solution for $(2.7)$ is

$$
\begin{aligned}
Y^{*} & =G^{*}\left(1-F_{\rho}(\rho)\right)=G_{+}^{*}\left(1-F_{\rho}(\rho)\right) \mathbf{1}_{z_{1}^{*}<1-F_{\rho}(\rho) \leq 1}-G_{-}^{*}\left(1-F_{\rho}(\rho)\right) \mathbf{1}_{0<1-F_{\rho}(\rho) \leq z_{1}^{*}} \\
& =G_{+}^{*}\left(1-F_{\rho}(\rho)\right) \mathbf{1}_{\rho<F_{\rho}^{-1}\left(1-z_{1}^{*}\right)}-G_{-}^{*}\left(1-F_{\rho}(\rho)\right) \mathbf{1}_{\rho \geq F_{\rho}^{-1}\left(1-z_{1}^{*}\right)} .
\end{aligned}
$$

Hence the optimal terminal wealth is

$$
X^{*}=Y^{*}+k=G_{+}^{*}\left(1-F_{\rho}(\rho)\right) \mathbf{1}_{\rho<F_{\rho}^{-1}\left(1-z_{1}^{*}\right)}-G_{-}^{*}\left(1-F_{\rho}(\rho)\right) \mathbf{1}_{\rho \geq F_{\rho}^{-1}\left(1-z_{1}^{*}\right)}+k .
$$

Remark 6.1 In this remark, we discuss the economical interpretation of the optimal wealth profile (6.1). Note that if we consider $k$ as a "reference point", then $G_{+}^{*}\left(1-F_{\rho}(\rho)\right) \mathbf{1}_{\rho<F_{\rho}^{-1}\left(1-z_{1}^{*}\right)}$ is the gain and $G_{-}^{*}\left(1-F_{\rho}(\rho)\right) \mathbf{1}_{\rho \geq F_{\rho}^{-1}\left(1-z_{1}^{*}\right)}$ is the loss. The optimal terminal wealth having a gain or a loss is determined by the terminal state density price being lower or higher than a single threshold $F_{\rho}^{-1}\left(1-z_{1}^{*}\right)$, where $z_{1}^{*}$ is optimal for problem (4.1).

After some simple calculations, we find that the efficient frontier of problem (2.2) is

$$
\begin{array}{r}
\widetilde{V}[x(T)]=\widetilde{V}\left[X^{*}\right]=\lambda_{+} a_{+}^{*}-\mu_{+} y_{+}^{*}+\lambda_{-} a_{+}^{*}-\mu_{-}\left(y_{+}^{*}-y_{0}\right) \\
=\lambda_{+} a_{+}^{*}-\mu_{+} y_{+}^{*}+\lambda_{-} a_{+}^{*}-\mu_{-} y_{+}^{*}+\mu_{-} x_{0}-\mu_{-} k \mathbb{E}(\rho),
\end{array}
$$

where $\left(\lambda_{+}, \mu_{+}\right),\left(\lambda_{-}, \mu_{-}\right)$are the unique Lagrange multipliers for the positive and negative part problems respectively. Note that $\lambda_{+}, \mu_{+}$, and $\lambda_{-}, \mu_{-}$depend on $\left(z_{1}^{*}, a_{+}^{*}, y_{+}^{*}\right)$, which are optimal values for problem (4.1). Moreover, $\lambda_{-}, \mu_{-}$depend on $k$.

In order to obtain the scheme of the efficient strategy from problem $(2.2)$, we let $w_{+}(z)$ be a concave probability distortion function, meaning that $w_{+}^{\prime}(z)$ is decreasing for $z \in(0,1)$ and $w_{+}^{\prime}(1-z)$ is increasing for $z \in(0,1)$, then $M_{+}(z)=\frac{F_{\rho}^{-1}(1-z)}{w_{+}^{\prime}(1-z)}$ is decreasing. It follows that $M_{+}(z)$, with $z \in\left(z_{1}, 1\right)$, is included in Case 2 with $\mathbb{E}_{+}^{z_{1}}(\rho) \in\left[M_{+}(1), M_{+}\left(z_{1}\right)\right)$ and $z_{0+}=z_{1}$, or equivalently, in Case 3 with $\mathbb{E}_{+}^{z_{1}}(\rho) \in\left[M_{+}(1), M_{+}\left(z_{1}\right)\right)$ and $z_{0+}=1$. 
Further, let $w_{-}(z)$ be a convex probability distortion function, then $w_{-}^{\prime}(z)$ is increasing for $z \in(0,1)$. Thus $M_{-}(z)=\frac{F_{\rho}^{-1}(1-z)}{w_{-}^{\prime}(z)}$ is decreasing. It follows that $M_{-}(z), z \in\left(0, z_{1}\right)$, is included in Case 2 with $\mathbb{E}_{-}^{z_{1}}(\rho) \in\left(M_{-}\left(z_{1}\right), M_{-}(0)\right]$ and $z_{0-}=0$, or equivalently, in Case 3 with $\mathbb{E}_{-}^{z_{1}}(\rho) \in\left[M_{-}\left(z_{1}\right), M_{-}(0)\right)$ and $z_{0-}=z_{1}$.

From this result, we know that there is no feasible solution for problem (2.11) and problem (2.12) if $a_{+} \mathbb{E}_{+}^{z_{1}}(\rho)<y_{0}+a_{+} \mathbb{E}_{-}^{z_{1}}(\rho)$ and $y_{+} \in\left(a_{+} \mathbb{E}_{+}^{z_{1}}(\rho), y_{0}+a_{+} \mathbb{E}_{-}^{z_{1}}(\rho)\right)$. So the optimal values of $\left(z_{1}, a_{+}, y_{+}\right)$for problem (4.1) must satisfy $y_{0}+a_{+} \mathbb{E}_{-}^{z_{1}}(\rho)<y_{+}<a_{+} \mathbb{E}_{+}^{z_{1}}(\rho)$ and then $\mu_{+}>0, \mu_{-}<$ 0 . The optimal solutions for problems (2.11) and (2.12) are $G_{+}^{*}(z)=\left[\lambda_{+}-\mu_{+} M_{+}(z)\right]^{+} \mathbf{1}_{z_{1}<z \leq 1}$ and $G_{-}^{*}(z)=\left[\lambda_{-}-\mu_{-} M_{-}(z)\right]^{+} \mathbf{1}_{0<z \leq z_{1}}$. Consequently, the optimal solution for problem (2.8) is

$$
G^{*}(z)=G_{+}^{*}(z)-G_{-}^{*}(z)=\left[\lambda_{+}-\mu_{+} M_{+}(z)\right]^{+} \mathbf{1}_{z_{1}<z \leq 1}-\left[\lambda_{-}-\mu_{-} M_{-}(z)\right]^{+} \mathbf{1}_{0<z \leq z_{1}} .
$$

Moreover, the optimal terminal cash flow is

$$
\begin{aligned}
X^{*} & =k+\left[\lambda_{+}-\mu_{+} M_{+}\left(1-F_{\rho}(\rho)\right)\right]^{+} \mathbf{1}_{\rho<F_{\rho}^{-1}\left(1-z_{1}^{*}\right)}-\left[\lambda_{-}-\mu_{-} M_{-}\left(1-F_{\rho}(\rho)\right)\right]^{+} \mathbf{1}_{\rho \geq F_{\rho}^{-1}\left(1-z_{1}^{*}\right)} \\
& =k+\left[\lambda_{+}-\mu_{+} \frac{\rho}{w_{+}^{\prime}\left(F_{\rho}(\rho)\right)}\right]^{+} \mathbf{1}_{\rho<c^{*}}-\left[\lambda_{-}-\mu_{-} \frac{\rho}{w_{-}^{\prime}\left(1-F_{\rho}(\rho)\right)}\right]^{+} \mathbf{1}_{\rho \geq c^{*}}
\end{aligned}
$$

where $c^{*}:=F_{\rho}^{-1}\left(1-z_{1}^{*}\right)$.

Recall that the optimal portfolio $\pi^{*}(\cdot)$ is the replicating portfolio for the claim $x(T)=X^{*}$. Let $\left(x_{1}(\cdot), \pi_{1}(\cdot)\right)$ replicate $1,\left(x_{2}(\cdot), \pi_{2}(\cdot)\right)$ replicate $\left[\lambda_{+}-\mu_{+} \frac{\rho}{w_{+}^{\prime}\left(F_{\rho}(\rho)\right)}\right]^{+} \mathbf{1}_{\rho<c^{*}}$, and $\left(x_{3}(\cdot), \pi_{3}(\cdot)\right)$ replicate $\left[\lambda_{-}-\mu_{-} \frac{\rho}{w_{-}^{\prime}\left(1-F_{\rho}(\rho)\right)}\right]^{+} \mathbf{1}_{\rho \geq c^{*}}$. Then, the claim is that $x(t)=k x_{1}(t)+x_{2}(t)-x_{3}(t)$, and the replicating portfolio for the claim $x(t)$, is $\pi(t)\left(=\pi^{*}(t)\right)=k \pi_{1}(t)+\pi_{2}(t)-\pi_{3}(t)$ (see Bielecki et al., 2005).

To give the explicit form of the claim, $x(t)$, and the strategy, $\pi(t)$, we assume that all market parameters are deterministic and time-invariant, i.e., $r(\cdot) \equiv r, B(\cdot) \equiv B, \sigma(\cdot) \equiv \sigma$ and $\theta(\cdot) \equiv \theta$. In this case, $\rho(t, T):=\frac{\rho(T)}{\rho(t)}$ follows a log-normal distribution with the parameters $\left(\tilde{\eta}_{t}, \tilde{\sigma}_{t}\right)$, where

$$
\tilde{\eta}_{t}:=-\left(r+\frac{\theta^{2}}{2}\right)(T-t) \text { and } \tilde{\sigma}_{t}^{2}:=\theta^{2}(T-t)
$$

$\rho(T)=\rho(0, T)$ follows a log-normal distribution with the parameters $\left(\tilde{\eta}_{0}, \tilde{\sigma}_{0}\right)$. Let $\Phi(\cdot)$ and $\phi(\cdot)$ be the cumulative distribution function and the density function of the standard normal distribution, respectively.

Using (2.4), we have

$$
\begin{aligned}
& x_{1}(t)=e^{r(t-T)}, \\
& x_{2}(t)= \mathbb{E}\left\{\rho(t, T)\left[\lambda_{+}-\mu_{+} \frac{\rho}{w_{+}^{\prime}\left(F_{\rho}(\rho)\right)}\right]^{+} \mathbf{1}_{\rho<c^{*}} \mid \mathcal{F}_{t}\right\} \\
&=\mathbb{E}\left\{\rho(t, T)\left[\lambda_{+}-\mu_{+} \frac{\rho(t, T) \rho(t)}{w_{+}^{\prime}\left(F_{\rho(T)}(\rho(t, T) \rho(t))\right)}\right]^{+} \mathbf{1}_{\rho(t, T)<\frac{c^{*}}{\rho(t)}} \mid \mathcal{F}_{t}\right\} \\
&=\int_{0}^{\frac{c^{*}}{\rho(t)}} y\left[\lambda_{+}-\mu_{+} \frac{y \rho(t)}{w_{+}^{\prime}\left(\Phi\left(\frac{\ln y+\ln \rho(t)-\tilde{\eta}_{0}}{\tilde{\sigma}_{0}}\right)\right)}\right]^{+} d \Phi\left(\frac{\ln y-\tilde{\eta}_{t}}{\tilde{\sigma}_{t}}\right) \\
&:=g_{2}(t, \rho(t)) .
\end{aligned}
$$


and

$$
\begin{aligned}
x_{3}(t) & =\mathbb{E}\left\{\rho(t, T)\left[\lambda_{-}-\mu_{-} \frac{\rho}{w_{-}^{\prime}\left(1-F_{\rho}(\rho)\right)}\right]^{+} \mathbf{1}_{\rho \geq c^{*}} \mid \mathcal{F}_{t}\right\} \\
& =\mathbb{E}\left\{\rho(t, T)\left[\lambda_{-}-\mu_{-} \frac{\rho(t, T) \rho(t)}{w_{-}^{\prime}\left(1-F_{\rho(T)}(\rho(t, T) \rho(t))\right)}\right]^{+} \mathbf{1}_{\left.\rho(t, T) \geq \frac{c^{*}}{\rho(t)} \mid \mathcal{F}_{t}\right\}}\right]^{+} d \Phi\left(\frac{\ln y-\tilde{\eta}_{t}}{\tilde{\sigma}_{t}}\right) \\
& =\int_{\frac{c^{*}}{\rho(t)}}^{+\infty} y\left[\lambda_{-}-\mu_{-} \frac{y(t)}{w_{-}^{\prime}\left(1-\Phi\left(\frac{\ln y+\ln \rho(t)-\tilde{\eta}_{0}}{\tilde{\sigma}_{0}}\right)\right)}\right] \\
& :=g_{3}(t, \rho(t)) .
\end{aligned}
$$

It is well-known (e.g. Bielecki et al. (2005)) that the replicating portfolio of $x_{1}(t), x_{2}(t), x_{3}(t)$ are

$$
\begin{gathered}
\pi_{1}(t)=0, \\
\pi_{2}(t)=-\left(\sigma \sigma^{\top}\right)^{-1} B \frac{\partial g_{2}(t, \rho)}{\partial \rho} \rho(t), \\
\pi_{3}(t)=-\left(\sigma \sigma^{\top}\right)^{-1} B \frac{\partial g_{3}(t, \rho)}{\partial \rho} \rho(t) .
\end{gathered}
$$

This allows us to obtain the explicit form of $\left(x(t), \pi^{*}(t)\right)$.

Remark 6.2 In the above paragraphs, we conclude that the efficient frontier of problem (2.2) is

$$
\widetilde{V}[x(T)]=\lambda_{+} a_{+}^{*}-\mu_{+} y_{+}^{*}+\lambda_{-} a_{+}^{*}-\mu_{-} y_{+}^{*}+\mu_{-} x_{0}-\mu_{-} k \mathbb{E}(\rho) .
$$

It seems that the variance is a linear function of the target return $k$. But actually, when we can derive the explicit solution of $\lambda_{-}$and $\mu_{-}$(such as the cases in Theorem 4.1), we have

$$
\mu_{-}=\frac{\frac{y_{+}-y_{0}-a_{+} \mathbb{E}_{-}^{z_{1}}(\rho)}{\int_{0}^{z_{1}} w_{-}^{\prime}(z) d z}}{\left\{\int_{z_{-}^{*}}^{z_{1}}\left[M_{-}\left(z_{-}^{*}\right)-M_{-}(z)\right] \frac{w_{-}^{\prime}(z)}{\int_{0}^{z_{1}} w_{-}^{\prime}(x) d x} d z\right\}^{2}-\int_{z_{-}^{*}}^{z_{1}}\left[M_{-}\left(z_{-}^{*}\right)-M_{-}(z)\right]^{2} \frac{w_{-}^{\prime}(z)}{\int_{0}^{z_{1}} w_{-}^{\prime}(x) d x} d z}
$$

and

$$
\lambda_{-}=\frac{a_{+}}{\int_{0}^{z_{1}} w_{-}^{\prime}(z) d z}+\mu_{-} \mathbb{E}_{-}^{z_{1}}(\rho)
$$

in (4.6). Recall that

$$
y_{0}:=x_{0}-k \mathbb{E}(\rho) .
$$

So both $\lambda_{-}$and $\mu_{-}$are linear in $k$. Then the variance is a quadratic function of $k$.

If we can derive the explicit solution of $\lambda_{-}$and $\mu_{-}$, then both $\lambda_{-}$and $\mu_{-}$are linear in $k$. We may conjecture that the variance may be a quadratic function of $k$ in general. 


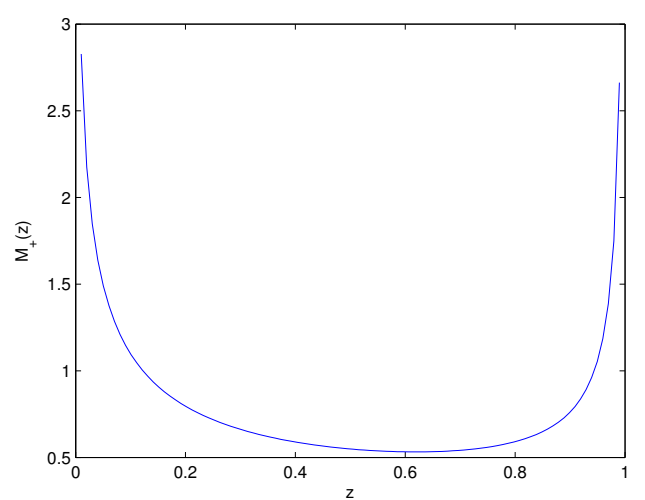

Fig. 7. $M_{+}(z)$ in Section 7

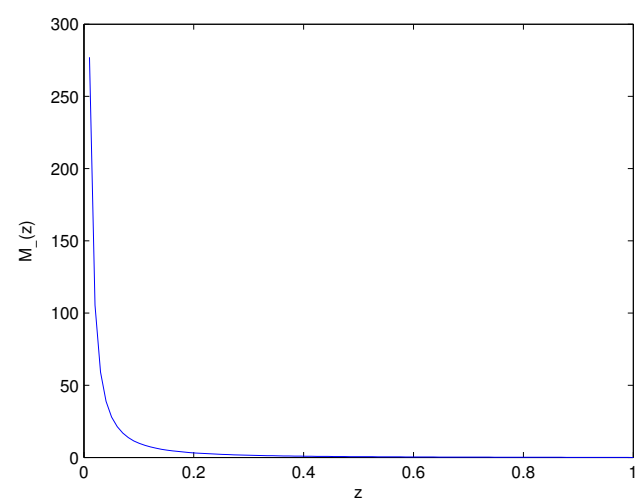

Fig. 8. $M_{-}(z)$ in Section 7

\section{$7 \quad$ Numerical examples}

In this section, we give some numerical examples to illustrate our results according to the result in Section 4. We assume that $\rho$ follows a log-normal distribution with the parameters $\left(\tilde{\eta}, \tilde{\sigma}^{2}\right)$. We take $w_{+}(z)=z^{\gamma}$ for $\gamma=2$, it is not difficult to verify that $M_{+}(z)=\frac{F_{\rho}^{-1}(1-z)}{w_{+}^{\prime}(1-z)}$ is included in Case 3. Take $\tilde{\eta}=-0.6, \tilde{\sigma}=1, x_{0}=0.5, T=1$, and then we have $\mathbb{E}(\rho)=0.9048$. Using MATLAB, we obtain the following numerical results.

First of all, we show $M_{+}(z)$ in Figure 7 . We find that $M_{+}(z)$ attains its minimum value at $z_{0}=0.6162$, and $M_{+}(0.6162)=0.5320$.

Next we give the numerical results of $v_{+}\left(z_{1}, a_{+}, y_{+}\right)$, which is the optimal value for the positive part problem (2.11). According to Theorem $4.1 \mathrm{i}$ ), we know that if (4.2) is satisfied, the optimal solution $G_{+}^{*}(z)$ and the optimal value $v_{+}\left(z_{1}, a_{+}, y_{+}\right)$are given in Theorem 4.1. In the following, we assume that (4.2) is satisfied.

We denote the right side of equation (4.2) as

$$
R\left(z_{1}, z_{+}^{*}\right):=\frac{\int_{z_{+}^{*}}^{1} M_{+}(z)\left[M_{+}(z)-M_{+}\left(z_{+}^{*}\right)\right] \frac{w_{+}^{\prime}(1-z)}{\int_{z_{1}}^{1} w_{+}^{\prime}(1-x) d x} d z}{\int_{z_{+}^{*}}^{1}\left[M_{+}(z)-M_{+}\left(z_{+}^{*}\right)\right] \frac{w_{+}^{\prime}(1-z)}{\int_{z_{1}}^{1} w_{+}^{\prime}(1-x) d x} d z} .
$$

We choose values for $\left(z_{1}, a_{+}, y_{+}\right)$that satisfy condition (4.2) in Theorem $\left.4.1 \mathrm{i}\right)$. We show the numerical values of $z_{1}, z_{+}^{*}, \mathbb{E}_{+}^{z_{1}}(\rho)$ and $R\left(z_{1}, z_{+}^{*}\right)$ in Table 1 . Note that $z_{+}^{*}$ is given in (3.6). $\mathbb{E}_{+}^{z_{1}}(\rho)=\frac{\int_{z_{1}}^{1} F_{\rho}^{-1}(1-z) d z}{\int_{z_{1}}^{1} w_{+}^{\prime}(1-z) d z}$ and $R\left(z_{1}, z_{+}^{*}\right)$ are determined by $z_{1}$. When $z_{1} \geq z_{0}=0.6162$, we get $z_{+}^{*}=z_{1}$, which is determined by the shape of $M_{+}(z)$ and the definition of $z_{+}^{*}$. From Table 1, we can see that: when the value of $z_{1}$ increases, the values of $z_{+}^{*}, \mathbb{E}_{+}^{z_{1}}(\rho)$ and $R\left(z_{1}, z_{+}^{*}\right)$ initially decrease, but later start to increase after reaching the minimum value at some $z \in\left[z_{1}, 1\right)$.

Based on condition (4.2) and $v_{+}\left(z_{1}, a_{+}, y_{+}\right)$given by (4.4) in Theorem $4.1 \mathrm{i}$ ), we are ready to give the numerical values of $v_{+}\left(z_{1}, a_{+}, y_{+}\right)$. We take $z_{1}=0.1,0.3$ and 0.5 , respectively, and then we give the corresponding $v_{+}\left(z_{1}, a_{+}, y_{+}\right)$in Tables 2,3 and 4 , respectively.

- The numerical solution of $v_{+}\left(z_{1}, a_{+}, y_{+}\right)$with $z_{1}=0.1$ is placed in Table 2. First, we keep $a_{+}=1$ and 0.5, respectively, and see what happens when we alter the value of $y_{+}$. From Table 2, we see that, for a fixed $z_{1}$ and $a_{+}, v_{+}\left(z_{1}, a_{+}, y_{+}\right)$increases as $y_{+}$increases. 
Second, we keep $y_{+}=1$ and 0.5 , respectively, and see what happens when we alter the value of $a_{+}$. We find that, for a fixed $z_{1}$ and $y_{+}, v_{+}\left(z_{1}, a_{+}, y_{+}\right)$decreases as $a_{+}$increases. $v_{+}\left(z_{1}, a_{+}, y_{+}\right)$attains a very small value when $a_{+}$is big and $y_{+}$is small. For example, when $a_{+}=0.0145$ and $y_{+}=0.01$, we have $v_{+}(0.1,0.0145,0.01)=2.5771 \times 10^{-4}$.

- We show the numerical solution of $v_{+}\left(z_{1}, a_{+}, y_{+}\right)$with $z_{1}=0.3$ in Table 3. Follow the same steps as above, then we get similar conclusions as we did above. $v_{+}\left(z_{1}, a_{+}, y_{+}\right)$attains a very small value when $a_{+}$is big and $y_{+}$is small, for example, $v_{+}(0.3,0.0170,0.01)=$ $5.8421 \times 10^{-4}$.

- We provide the numerical solution of $v_{+}\left(z_{1}, a_{+}, y_{+}\right)$with $z_{1}=0.5$ in Table 4 . Follow the same steps as above, then we get the similar conclusions as we did above. $v_{+}\left(z_{1}, a_{+}, y_{+}\right)$attains a very small value when $a_{+}$is big and $y_{+}$is small, for example, $v_{+}(0.5,0.0174,0.01)=$ 0.0012 .

Finally, we give the numerical solution of the efficient frontier. We assume that $w_{-}(z)$ has the form $w_{-}(z)=z^{\gamma}$ for $\gamma=2$. We can verify that $M_{-}(z)=\frac{F_{\rho}^{-1}(1-z)}{w_{-}^{\prime}(z)}$ is strictly decreasing

Table 1: $z_{1}, z_{+}^{*}, \mathbb{E}_{+}^{z_{1}}(\rho), R\left(z_{1}, z_{+}^{*}\right), \mathbb{E}_{-}^{z_{1}}(\rho)$

\begin{tabular}{|c|c|c|c|c|c|c|c|c|c|}
\hline$z_{1}$ & 0.1 & 0.2 & 0.3 & 0.4 & 0.5 & 0.6 & 0.7 & 0.8 & 0.9 \\
\hline$z_{+}^{*}$ & 0.8686 & 0.8207 & 0.7741 & 0.7266 & 0.6782 & 0.6284 & 0.7000 & 0.8000 & 0.9000 \\
\hline $\mathbb{E}_{+}^{z_{1}}(\rho)$ & 0.6867 & 0.6198 & 0.5866 & 0.5724 & 0.5741 & 0.5935 & 0.6396 & 0.7398 & 1.0154 \\
\hline$R\left(z_{1}, z_{+}^{*}\right)$ & 1.5380 & 1.3298 & 1.2067 & 1.1275 & 1.0807 & 1.0646 & 1.0980 & 1.2681 & 1.7620 \\
\hline $\mathbb{E}_{-}^{z_{1}}(\rho)$ & 33.9467 & 12.5555 & 6.8549 & 4.4042 & 3.0967 & 2.3057 & 1.7858 & 1.4232 & 1.1583 \\
\hline
\end{tabular}

Table 2: $v_{+}\left(z_{1}, a_{+}, y_{+}\right)$with $z_{1}=0.1, z_{+}^{*}=0.8686, \mathbb{E}_{+}^{z_{1}}(\rho)=0.6867, R\left(z_{1}, z_{+}^{*}\right)=1.5380$

\begin{tabular}{|c|c|c|c|c|c|c|c|}
\hline$y_{+}$ & 0.7 & 0.8 & 0.9 & 1.0 & 1.1 & 1.2 & 1.3 \\
\hline$v_{+}\left(0.1,1, y_{+}\right)$ & 1.2820 & 5.4052 & 16.0145 & 33.1100 & 56.6916 & 86.7595 & 123.3135 \\
\hline \hline$y_{+}$ & 0.35 & 0.40 & 0.45 & 0.50 & 0.55 & 0.60 & 0.65 \\
\hline$v_{+}\left(0.1,0.5, y_{+}\right)$ & 0.3205 & 1.3513 & 4.0036 & 8.2775 & 14.1729 & 21.6899 & 30.8284 \\
\hline \hline$a_{+}$ & 0.8 & 0.9 & 1.0 & 1.1 & 1.2 & 1.3 & 1.4 \\
\hline$v_{+}\left(0.1, a_{+}, 1\right)$ & 66.7045 & 48.3669 & 33.1100 & 20.9337 & 11.8381 & 5.8232 & 2.8889 \\
\hline \hline$a_{+}$ & 0.40 & 0.45 & 0.50 & 0.55 & 0.60 & 0.65 & 0.70 \\
\hline$v_{+}\left(0.1, a_{+}, 0.5\right)$ & 16.6761 & 12.0917 & 8.2775 & 5.2334 & 2.9595 & 1.4558 & 0.7222 \\
\hline
\end{tabular}

Table 3: $v_{+}\left(z_{1}, a_{+}, y_{+}\right)$with $z_{1}=0.3, z_{+}^{*}=0.7741, \mathbb{E}_{+}^{z_{1}}(\rho)=0.5866, R\left(z_{1}, z_{+}^{*}\right)=1.2067$

\begin{tabular}{|c|c|c|c|c|c|c|c|}
\hline$y_{+}$ & 0.6 & 0.7 & 0.8 & 0.9 & 1.0 & 1.1 & 1.2 \\
\hline$v_{+}\left(0.3,1, y_{+}\right)$ & 2.0648 & 5.0635 & 12.7699 & 25.1840 & 42.3057 & 64.1351 & 90.6721 \\
\hline \hline$y_{+}$ & 0.30 & 0.35 & 0.40 & 0.45 & 0.50 & 0.55 & 0.60 \\
\hline$v_{+}\left(0.3,0.5, y_{+}\right)$ & 0.5162 & 1.2659 & 3.1925 & 6.2960 & 10.5764 & 16.0338 & 22.6680 \\
\hline \hline$a_{+}$ & 1.1 & 1.2 & 1.3 & 1.4 & 1.5 & 1.6 & 1.7 \\
\hline$v_{+}\left(0.3, a_{+}, 1\right)$ & 32.1206 & 23.5942 & 16.7265 & 11.5174 & 7.9670 & 6.0752 & 5.8421 \\
\hline \hline$a_{+}$ & 0.55 & 0.60 & 0.65 & 0.70 & 0.75 & 0.80 & 0.85 \\
\hline$v_{+}\left(0.3, a_{+}, 0.5\right)$ & 8.0302 & 5.8986 & 4.1816 & 2.8793 & 1.9917 & 1.5188 & 1.4605 \\
\hline
\end{tabular}


across $(0,1)$. We show $M_{-}(z)$ in Figure 8. According to Section 4.1, we know $\mu_{-} \geq 0$ when $a_{+} \mathbb{E}_{+}^{z_{1}}(\rho) \leq y_{0}+a_{+} \mathbb{E}_{-}^{z_{1}}(\rho)$ and $y_{+} \in\left[a_{+} \mathbb{E}_{+}^{z_{1}}(\rho), y_{0}+a_{+} \mathbb{E}_{-}^{z_{1}}(\rho)\right]$. So the optimal solution for problem (3.2) is $G_{-}(z) \equiv G$. Insert $G_{-}(z) \equiv G$ into the constraints in (2.12), then we have $y_{+}-y_{0}=a_{+} \mathbb{E}_{-}^{z_{1}}(\rho), G_{-}(z) \equiv G=\frac{a_{+}}{\int_{0}^{z_{1}} w_{-}^{\prime}(z) d z}$, and $v_{-}\left(z_{1}, a_{+}, y_{+}\right)=\frac{a_{+}^{2}}{\int_{0}^{z_{1}} w_{-}^{\prime}(z) d z}$. Recall that $y_{0}=x_{0}-k \mathbb{E}(\rho)(\leq 0)$, then we get

$$
\begin{aligned}
& \hat{v}\left(z_{1}, a_{+}\right):=v_{+}\left(z_{1}, a_{+}, y_{0}+a_{+} \mathbb{E}_{-}^{z_{1}}(\rho)\right)+v_{-}\left(z_{1}, a_{+}, y_{0}+a_{+} \mathbb{E}_{-}^{z_{1}}(\rho)\right) \\
& =\frac{\frac{\left[x_{0}-k \mathbb{E}(\rho)+a_{+} \mathbb{E}_{-}^{z_{1}}(\rho)-a_{+} \mathbb{E}_{+}^{z_{1}}(\rho)\right]^{2}}{\int_{z_{1}}^{1} w_{+}^{\prime}(1-z) d z}}{\int_{z_{+}^{*}}^{1}\left[M_{+}\left(z_{+}^{*}\right)-M_{+}(z)\right]^{2} \frac{w_{+}^{\prime}(1-z)}{\int_{z_{1}}^{1} w_{+}^{\prime}(1-x) d x} d z-\left\{\int_{z_{+}^{*}}^{1}\left[M_{+}\left(z_{+}^{*}\right)-M_{+}(z)\right] \frac{w_{+}^{\prime}(1-z)}{\int_{z_{1}}^{1} w_{+}^{\prime}(1-x) d x} d z\right\}^{2}} \\
& +\frac{a_{+}^{2}}{\int_{z_{1}}^{1} w_{+}^{\prime}(1-z) d z}+\frac{a_{+}^{2}}{\int_{0}^{z_{1}} w_{-}^{\prime}(z) d z} .
\end{aligned}
$$

In the second step, we need to choose a $z_{1}$ and $a_{+}$to minimize $\hat{v}\left(z_{1}, a_{+}\right)$. Because we have the explicit form of $\hat{v}\left(z_{1}, a_{+}\right)$, it is not difficult to solve the problem in second step. We denote the optimal $\left(z_{1}, a_{+}\right)$as $\left(z_{1}^{*}, a_{+}^{*}\right)$. So the efficient frontier is

$$
\begin{aligned}
& \widetilde{V}(x(T))=\hat{v}\left(z_{1}^{*}, a_{+}^{*}\right)=\frac{\left(a_{+}^{*}\right)^{2}}{\int_{z_{1}^{*}}^{1} w_{+}^{\prime}(1-z) d z}+\frac{\left(a_{+}^{*}\right)^{2}}{\int_{0}^{z_{1}^{*}} w_{-}^{\prime}(z) d z} \\
& +\frac{\frac{\left[x_{0}-k \mathbb{E}(\rho)+a_{+}^{*} \mathbb{E}_{-}^{z_{1}}(\rho)-a_{+}^{*} \mathbb{E}_{+}^{z_{1}}(\rho)\right]^{2}}{\int_{z_{1}^{*}}^{1} w_{+}^{\prime}(1-z) d z}}{\int_{z_{+}^{*}}^{1}\left[M_{+}\left(z_{+}^{*}\right)-M_{+}(z)\right]^{2} \frac{w_{+}^{\prime}(1-z)}{\int_{z_{1}^{*}}^{1} w_{+}^{\prime}(1-x) d x} d z-\left\{\int_{z_{+}^{*}}^{1}\left[M_{+}\left(z_{+}^{*}\right)-M_{+}(z)\right] \frac{w_{+}^{\prime}(1-z)}{\int_{z_{1}^{*}}^{1} w_{+}^{\prime}(1-x) d x} d z\right\}^{2}} .
\end{aligned}
$$

The efficient frontier when there is no probability distortion has been obtained in Zhou and Li (2000) as

$$
V(x(T)):=\operatorname{Var}(x(T))=\frac{1}{2.71828-1}\left[\mathbb{E} x(T)-0.5 * 2.71828^{0.1}\right]^{2} .
$$

Both (7.2) and (7.3) are plotted on the same plane; see Figure 9.

Note that the efficient frontier in our example, i.e., when the probability distortion functions have forms $w_{+}(z)=z^{\gamma}$ and $w_{-}(z)=z^{\gamma}$ for some $\gamma>1$, is also the quadratic function of the

Table 4: $v_{+}\left(z_{1}, a_{+}, y_{+}\right)$with $z_{1}=0.5, z_{+}^{*}=0.6782, \mathbb{E}_{+}^{z_{1}}(\rho)=0.5741, R\left(z_{1}, z_{+}^{*}\right)=1.0807$

\begin{tabular}{|c|c|c|c|c|c|c|c|}
\hline$y_{+}$ & 0.58 & 0.66 & 0.74 & 0.82 & 0.90 & 0.98 & 1.06 \\
\hline$v_{+}\left(0.5,1, y_{+}\right)$ & 3.9686 & 5.5298 & 9.7914 & 16.7535 & 26.4161 & 38.7792 & 53.8427 \\
\hline \hline$y_{+}$ & 0.29 & 0.33 & 0.37 & 0.41 & 0.45 & 0.49 & 0.53 \\
\hline$v_{+}\left(0.5,0.5, y_{+}\right)$ & 0.9922 & 1.3824 & 2.4478 & 4.1884 & 6.6040 & 9.6948 & 13.4607 \\
\hline \hline$a_{+}$ & 1.0 & 1.1 & 1.2 & 1.3 & 1.4 & 1.5 & 1.6 \\
\hline$v_{+}\left(0.5, a_{+}, 1\right)$ & 42.2919 & 33.4988 & 26.1740 & 20.3174 & 15.9290 & 13.0089 & 11.5571 \\
\hline \hline$a_{+}$ & 0.50 & 0.55 & 0.60 & 0.65 & 0.70 & 0.75 & 0.80 \\
\hline$v_{+}\left(0.5, a_{+}, 0.5\right)$ & 10.5730 & 8.3747 & 6.5435 & 5.0793 & 3.9823 & 3.2522 & 2.8893 \\
\hline
\end{tabular}




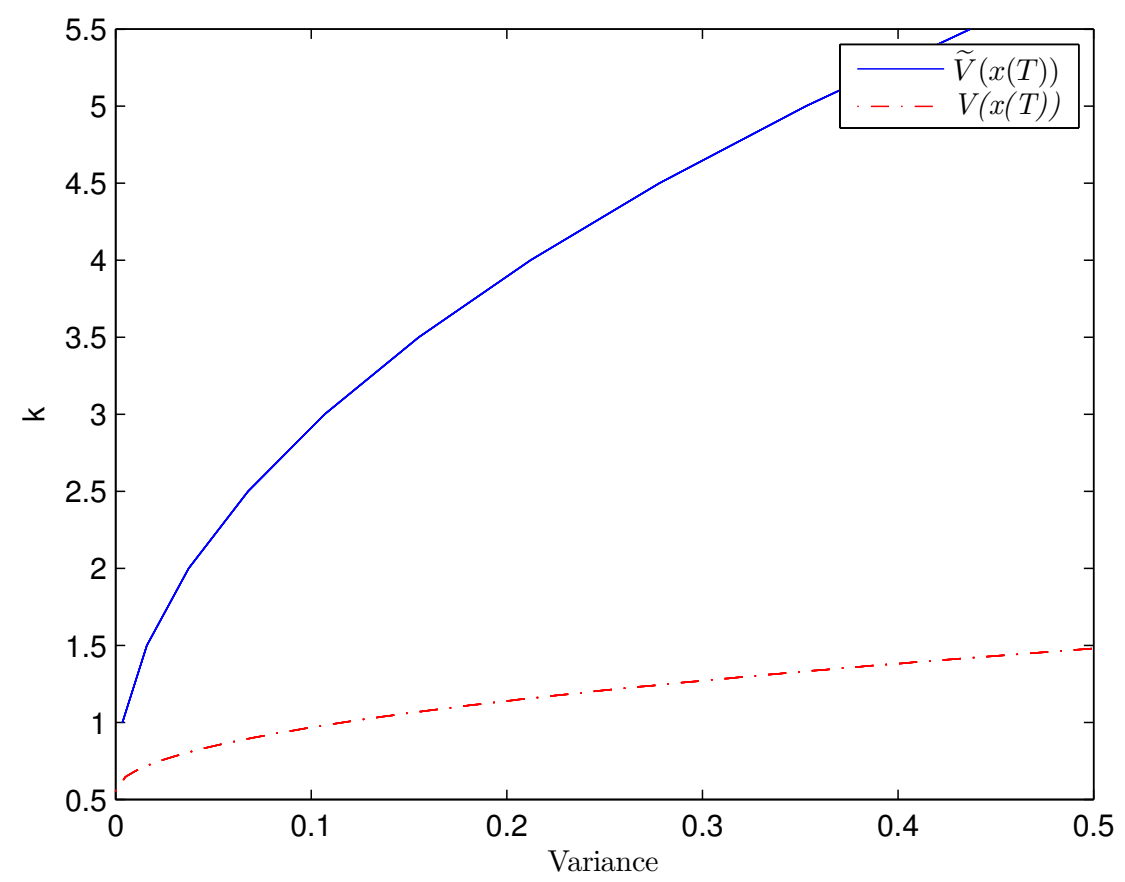

Fig. 9. The efficient frontier in Section 7

terminal distorted expectation, $k$, which is the same as the efficient frontier in the traditional mean-variance portfolio selection problem. From Figure 9 we can see that, the efficient frontier when there exists probability distortion rises above that when there is no probability distortion, i.e., the insurer can reach a lower risk when there exists probability distortion than that when there is no probability distortion with the same profit.

\section{Conclusion}

This paper formulates and studies the behavioral mean-variance portfolio selection problem. The probability distortion (or nonlinear expectation) destroys the time consistency, which is necessary for the dynamic programming approach, as well as the convexity, which is necessary for the convex duality approach. We turn the non-convex minimization problem into a convex minimization problem by changing the decision variable and performing a series of transformations. Then we use the Lagrange method to solve the convex minimization problem.

To further extend our solution scheme, it will be of interest to study the following problems:

First, in order to reflect the characteristics of the real financial market more exactly, one could build a more complex but real mathematical model. For example, we can investigate the behavioral mean-variance portfolio selection problems in continuous-time under the constraint that short-selling of stocks is not allowed. On the other hand, we would consider the behavioral continuous-time mean-variance portfolio selection with a bankruptcy prohibition, i.e., the wealth process under any admissible trading strategy is not allowed to be below zero at any time.

Second, because the problem of optimal investment-reinsurance for an insurer has attracted more and more attention in the past two decades, we could try to introduce the behavioral finance to the insurance company. Then we could consider the optimal behavioral mean-variance 
investment-reinsurance problem for an insurer.

\section{Appendix A: The proof of Lemma 3.2}

\section{Proof.}

i) Assume $M_{+}(z)$ is in Case 1. The result of $M_{+}(z)$ in Case 2 with $\mathbb{E}_{+}^{z_{1}}(\rho)>M_{+}\left(z_{1}\right)$ can be derived similarly. Because of $\mu_{+}<0, \lambda_{+}-\mu_{+} M_{+}(z)$ is included in Case 1. By Lemma 3.1, the optimal solution for (3.4) has the form

$$
G_{+}^{*}(z)=\left[\lambda_{+}-\mu_{+} M_{+}(z)\right] \mathbf{1}_{z_{1}<z \leq z_{2}}+\left[\lambda_{+}-\mu_{+} M_{+}\left(z_{2}\right)\right] \mathbf{1}_{z_{2}<z \leq 1},
$$

where $z_{2}$ is to be determined. Then problem (3.4) reduces to

$$
\min _{z_{2} \in\left[\bar{z}_{0+}, z_{0+}\right]} \mu_{+}^{2} \int_{z_{2}}^{1} w_{+}^{\prime}(1-z)\left[M_{+}\left(z_{2}\right)-M_{+}(z)\right]^{2} d z .
$$

where $\bar{z}_{0+} \in\left(z_{1}, z_{0+}\right)$ is the value at which $M_{+}\left(\bar{z}_{0+}\right)=M_{+}(1)$.

Denote

$$
N\left(z_{2}\right)=\int_{z_{2}}^{1} w_{+}^{\prime}(1-z)\left[M_{+}\left(z_{2}\right)-M_{+}(z)\right]^{2} d z, z_{2} \in\left[\bar{z}_{0+}, z_{0+}\right]
$$

we aim to find the minimizer of $N\left(z_{2}\right)$ over $\left[\bar{z}_{0+}, z_{0+}\right]$. Notice that

$$
N^{\prime}\left(z_{2}\right)=2 M_{+}^{\prime}\left(z_{2}\right) \int_{z_{2}}^{1} w_{+}^{\prime}(1-z)\left[M_{+}\left(z_{2}\right)-M_{+}(z)\right] d z=2 M_{+}^{\prime}\left(z_{2}\right) n\left(z_{2}\right),
$$

where

$$
n\left(z_{2}\right)=\int_{z_{2}}^{1} w_{+}^{\prime}(1-z)\left[M_{+}\left(z_{2}\right)-M_{+}(z)\right] d z .
$$

And $M_{+}^{\prime}\left(z_{2}\right)>0, z_{2} \in\left[\bar{z}_{0+}, z_{0+}\right]$. Thanks to the shape of $M(\cdot)$, we know that

$$
\begin{aligned}
& n\left(\bar{z}_{0+}\right)=\int_{\bar{z}_{0+}}^{1} w_{+}^{\prime}(1-z)\left[M_{+}\left(\bar{z}_{0+}\right)-M_{+}(z)\right] d z<0, \\
& n\left(z_{0+}\right)=\int_{z_{0+}}^{1} w_{+}^{\prime}(1-z)\left[M_{+}\left(z_{0+}\right)-M_{+}(z)\right] d z>0, \\
& n^{\prime}\left(z_{2}\right)=\int_{z_{2}}^{1} w_{+}^{\prime}(1-z) M_{+}^{\prime}\left(z_{2}\right) d z>0, z_{2} \in\left[\bar{z}_{0+}, z_{0+}\right] .
\end{aligned}
$$

So, there exists a unique $z_{+}^{*} \in\left[\bar{z}_{0+}, z_{0+}\right]$ which satisfies

$$
n\left(z_{+}^{*}\right)=\int_{z_{+}^{*}}^{1} w_{+}^{\prime}(1-z)\left[M_{+}\left(z_{+}^{*}\right)-M_{+}(z)\right] d z=0 .
$$

ii) The proof of ii) follows a similar argument to that of i). Then we finish the proof.

Acknowledgements Junna Bi is supported by National Natural Science Foundation of China (Grant No. 11571189) and 111 Project (B14019). Qingbin Meng is supported by National Natural Science Foundation of China (Grant No. 71302156). 


\section{References}

[1] Allais, M. (1953). Le comportement de l'homme rationnel devant le risque, critique des postulats et axiomes de l'ecole americaine. Econometrica, 21, 503-546.

[2] Bi, J., Zhong, Y., and Zhou, X. Y. (2013) Mean-semivariance portfolio selection under probability distortion. Stochastics-An International Journal of Probability and Stochastic Processes, 85(4), 604-619.

[3] Bielecki, T. R., Jin, H., Pliska, S. R. and Zhou, X. Y. (2005). Continuous-time meanvariance portfolio selection with bankrutcy probihition. Mathematical Finance, 15, 213-244.

[4] Björk, T., Murgoci, A. and Zhou, X. Y. (2014). Mean-variance portfolio optimization with state-dependent risk aversion. Mathematical Finance, 24(1), 1-24.

[5] Brocklesby, J. (2016). The what, the why and the how of behavioural operational researchAn invitation to potential sceptics. European Journal of Operational Research, 249, 796-805.

[6] Cillo, A. and Delquié, P. (2014). Mean-risk analysis with enhanced behavioral content. European Journal of Operational Research, 239, 764-775.

[7] Cui, X., Gao J., Li, X. and Li, D. (2014). Optimal multi-period mean-variance policy under no-shorting constraint. European Journal of Operational Research, 234, 459-468.

[8] Dang D.M. and Forsyth P.A. (2016). Better than pre-commitment mean-variance portfolio allocation strategies: A semi-self-financing Hamilton-Jacobi-Bellman equation approach. European Journal of Operational Research, 250(3), 827-841.

[9] Ellesberg, D. (1961). Risk, Ambiguity and the Savage Axioms. Quarterly Journal of Economics, 75, 643-669.

[10] Friedman, M. and Savage, L. J. (1948). The Utility Analysis of Choices Involving Risk. Journal of Political Economy, 56, 279-304.

[11] Gomes, F. J. (2005). Portfolio Choice and Trading Volume with Loss-Averse Investors. Journal of Business, 78(2), 675-706.

[12] He, X. D. and Zhou, X. Y. (2011). Portfolio Choice via Quantiles. Mathematical Finance, 21(2), 203-231.

[13] He, X. D., Hu S., Obłój J. and Zhou, X. Y. (2017). Technical Note-Path-Dependent and Randomized Strategies in Barberis' Casino Gambling Model. Operations Research, 65(1), 97-103.

[14] Jin, H. and Zhou, X.Y. (2008). Behavioral portfolio selection in continuous time. Mathematical Finance, 18, 385-426. Erratum. (2010). Mathematical Finance, 20, 521-525.

[15] Jin H. and Zhou X. Y. (2013). Greed, leverage, and potential losses: A prospect theory perspective. Mathematical Finance, 23(1), 122-142.

[16] Kahneman, D. and Tversky, A. (1979). Prospect theory: An analysis of decision under risk. Econometrica, 47, 263-291. 
[17] Karoui, N. EL., Peng, S. and Quenez, M. C. (1997). Backward stochastic differential equations in finance. Mathematical Finance, 7, 1-71.

[18] Levy, H. and Levy, M. (2004). Prospect Theory and Mean-variance Analysis. Review of Financial Studies, 17(4), 1015-1041.

[19] Li, X., Zhou, X. Y. and Lim A. E. B. (2002). Dynamic mean-variance portfolio selection with no-shorting constraints. SIAM Journal on Control and Optimization, 40, 1540-1555.

[20] Lioui A. and Poncet P. (2016). Understanding dynamic mean variance asset allocation. European Journal of Operational Research, 254(1), 320-337.

[21] Lopes, L. L. (1987). Between hope and fear: The psychology of risk. Advances in Experimental Social Psychology, 20, 255-295.

[22] Lopes, L. L. and Oden, G. C. (1999). The role of aspiration level in risk choice: A comparison of cumulative prospect theory and sp/a theory. Journal of Mathematical Psychology, 43, 286-313.

[23] Markowitz, H. (1952). Portfolio selection. The Journal of Finance, 7, 77-91.

[24] Markowitz, H. (2014). Mean-variance approximations to expected utility. European Journal of Operational Research, 234, 346-355.

[25] Mehra, R. and Prescott, E. C. (1985). The Equity Premium: A Puzzle. Journal of monetary Economics, 15, 145-161.

[26] Merton, R.C. (1972). An analytical derivation of the efficient portfolio frontier. Journal of Financial and Quantitative Analysis, 7, 1851-1872.

[27] Ray P. and Jenamani M. (2016). Mean-variance analysis of sourcing decision under disruption risk. European Journal of Operational Research, 250(2), 679-689.

[28] Tversky, A. and Kahneman, D. (1992). Advances in prospect theory: Cumulative representation of uncertainty. Journal of Risk and Uncertainty, 5, 297-323.

[29] Yaari, M. E. (1987). The dual theory of choice under risk. Econometrica, 55(1), 95-115.

[30] Yao, H., Li, Z. and Li, D. (2016). Multi-period mean-variance portfolio selection with stochastic interest rate and uncontrollable liability. European Journal of Operational Research, 252, 837-851.

[31] Zhou, X. Y. and Li, D. (2000). Continuous time mean-variance portfolio selection: A stochastic LQ framwork. Applied Mathematics and Optimization, 42, 19-33. 